\title{
1 Charge Transport via Delocalized States in Disordered Materials
}

\author{
Igor Zvyagin
}

Faculty of Physics, Moscow State University, 119899 Moscow, Russia

1.1 Introduction 2

1.2 Transport by Electrons in Extended States Far from the Mobility Edges 4

1.2.1 Weak-scattering theories 4

$\begin{array}{ll}1.2 .2 \text { Weak localization } & 10\end{array}$

1.2.3 Interaction effects 12

1.3 Scaling Theory of Localization 14

1.3.1 Main ideas of the scaling theory of localization $\quad 14$

1.3.2 The main equations of one-parameter scaling 15

1.3.3 Model solutions 18

1.3.4 Some predictions of the scaling theory 22

1.3.5 Minimum metallic conductivity 24

1.4 Extended-state Conduction in Three Dimensions 26

1.4.1 Activated conduction 26

1.4.2 Extended-state conduction near the
metal-insulator transition

1.5 Apparent Mobility Edge and Extended-state

1.5.1 Experimental studies of the mobility edge in low-mobility two-dimensional systems 33

1.5.2 Evidence for a true metal-insulator transition
in high-mobility two-dimensional systems

1.5.3 Evidence against a true metal-insulator
transition in two-dimensional systems

1.5.4 Temperature-dependent charge carrier scattering 38

1.6 Conclusions 43

References 44 


\subsection{INTRODUCTION}

The standard theory of electron transport phenomena in ideal crystalline semiconductors is based on the band theory that determines single-electron eigenstates (Bloch waves) and energies forming energy bands (with a quasi-continuous level distribution) separated by bandgaps. Static disorder modifies the electron wavefunctions and the energy spectrum of the system. A useful characteristic of the electron states is the density of states that can be applied to the description of both ordered solids and solids with arbitrarily strong disorder. In the presence of weak disorder, the eigenstates of a disordered material differ only slightly from the eigenstates of an ideal crystal and the density of states appears to be only slightly different from that of an ideal crystal. Therefore, the description of the electronic processes in weakly disordered crystalline solids may use the concept of almost free quasi-particles (Bloch waves) modified by the interaction with the random field and by the electron-electron interaction. Interacting electrons in extended states may be often described using the Fermiliquid approach, which assumes that a system of electrons is described by using a selfconsistent field that determines the properties of almost free quasi-particles, whose energy spectrum is a function of the interaction, and the distribution function. The wavefunctions remain extended over the entire sample and have a random component that may be described as a random phase variation of the electronic wave. Accordingly, transport in a system with weak disorder may be described using the basis of the unperturbed Bloch eigenstates and assuming that a random scattering potential (related to random static deviations of the potential in the crystal from periodicity, to lattice vibrations, etc.) induces transitions between these eigenstates. The corresponding theory is based on the Boltzmann transport equation, whose condition of applicability is

$$
\hbar / \tau<<\bar{\varepsilon},
$$

where $\tau$ is the quasi-momentum relaxation time and $\bar{\varepsilon}$ is the characteristic quasi-particle energy (equal to $k T$ for nondegenerate electron gas or to the Fermi energy $\varepsilon_{\mathrm{F}}$ for the degenerate case). Condition (1.1.1) allows one to combine the classical description of statistical properties of the gas of quasi-particles with quantum-mechanical treatment of individual scattering events. Condition (1.1.1) may be written in the alternative form

$$
\lambda<<l
$$

where $\lambda=\hbar / \bar{p}=\bar{k}^{-1}$ is the de Broglie wavelength of quasi-particles, $\bar{p}$ and $\bar{k}$ are their characteristic quasi-momentum and quasi-wave vector, $l=\bar{v} \tau$ is their mean free path, and $\bar{v}$ is the characteristic velocity of the quasi-particles.

In noncrystalline materials (amorphous solids and liquids), short-range order exists, even for strong structural disorder, and the electron energy spectrum is known to retain the characteristic features of the band spectrum: the regions of high electron density of states (corresponding to the allowed bands of the crystal) exist, separated by the regions where the density of states is lower (often by several orders of magnitude) corresponding to the

${ }^{1}$ Some of the problems discussed in this chapter are also treated in books [1-7] and reviews [8-11] and references therein. 
energy gaps of the band spectrum of the crystal. With increasing disorder, however, extended electron states inside the energy bands of the crystal may become localized (Anderson localization [12]); eventually, for sufficiently strong disorder, all electron states become localized. Localization implies that the envelope of the wave function decays exponentially away from some localization point $\boldsymbol{R}, \psi(r) \sim \exp (-|\mathbf{r}-\mathbf{R}| / \alpha)$, where $\alpha$ is the localization length. Mott has pointed out that the role of disorder can be different for states of different energies; for intermediate disorder, localized and extended states may coexist in the same system at different energies. Typically, the states in the middle of the band may remain extended (extended states are 'current-carrying', i.e., the average velocity in these states is nonzero); on the other hand, near the band edges, the states are localized (Figure 1.1). Following Mott, one may introduce the energy $\varepsilon_{\mathrm{c}}$, corresponding to the localization threshold that separates extended and localized states; this localization threshold is called the mobility edge [13]. Extended states above the mobility edge contribute to the DC current even at $T=0 \mathrm{~K}$ and if $\varepsilon_{\mathrm{F}}>\varepsilon_{\mathrm{c}}$, the conductivity $\sigma_{T \rightarrow 0 K}$ (and the resistivity $\rho_{T \rightarrow 0 K}$ ) remains finite. A finite conductivity at $T=0 \mathrm{~K}$ is a signature of the metallic state, whereas a vanishing conductivity (or a divergent resistivity $\rho_{T \rightarrow 0 K}$ ) of an infinite system as $T \rightarrow 0 \mathrm{~K}$ indicates that the system is an insulator. Therefore, a metal-insulator transition occurs as the Fermi level crosses the mobility edge $\varepsilon_{\mathrm{c}}$ passing from extended to localized states.

Mott has argued that the concept of the conduction band (the region of extended states above the mobility edge) may be used, even for materials with strong disorder (say, for amorphous semiconductors), the mobility edge $\varepsilon_{\mathrm{c}}$ playing the role of the conduction band edge. It should be noted that there is no singularity in the density of states at the mobility edge $\varepsilon_{\mathrm{c}}$. Likewise, one may introduce the concept of the mobility edge for the valence band $\varepsilon_{\mathrm{v}}$. The energy interval between $\varepsilon_{\mathrm{c}}$ and $\varepsilon_{\mathrm{v}}$ is called the mobility gap, and the states in the mobility gap are localized.

For extended states, once the electron scattering is weak, the Boltzmann equation makes it possible to calculate the transport coefficients. At finite temperatures, the usual expression

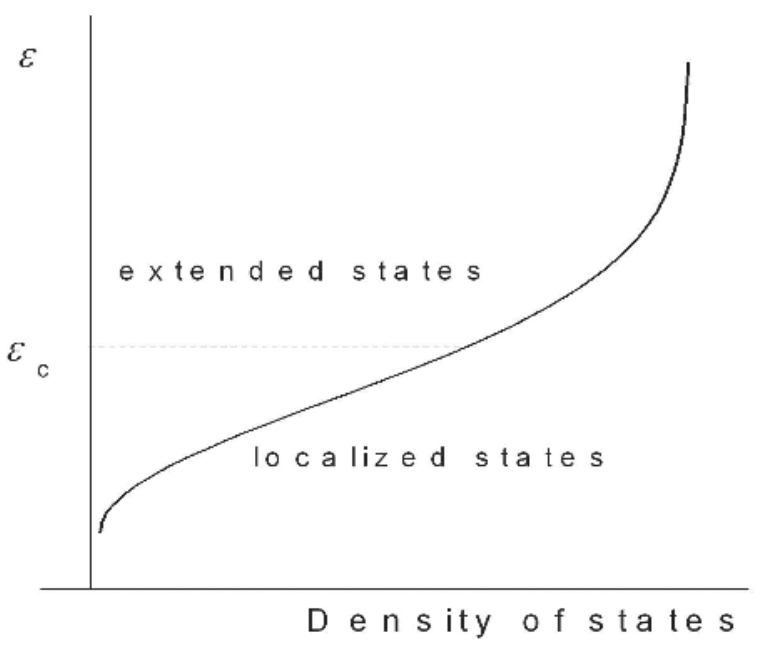

Figure 1.1 Density of states in a disordered solid (schematic); $\varepsilon_{\mathrm{c}}$ denotes the mobility edge that separates extended and localized states 
for the conductivity in the Boltzmann transport theory (linear with respect to the electric field) may be written [1] as

$$
\sigma=\int \sigma(\varepsilon)\left(-\frac{\partial f(\varepsilon)}{\partial \varepsilon}\right) \mathrm{d} \varepsilon
$$

where $f(\varepsilon)$ is the electron distribution function, $\sigma(\varepsilon)$ is the energy-dependent conductivity, and the integration in Equation (1.1.3) is performed over the extended states in the conduction band. (To be specific, in what follows, we usually speak about electrons and the conduction band; the modification for the case of holes and the valence band is straightforward.) Equation (1.1.3) immediately follows from the Boltzmann equation for elastic electron scattering; for inelastic scattering, $\sigma(\varepsilon)$ is only formally introduced by Equation (1.1.3) and must be determined from the corresponding transport theory. It follows from Equation (1.1.3) that in the region of extended states, $\left.\sigma\right|_{T \rightarrow 0 K}=\sigma\left(\varepsilon_{\mathrm{F}}\right)$, where $\varepsilon_{\mathrm{F}}$ is the Fermi level.

In the absence of long-range crystalline order, electronic states are different from Bloch states, quasi-momentum is no longer a good quantum number, the concept of the Brillouin zone cannot be used anymore, etc. Strictly speaking, for disordered (amorphous or liquid) metals and disordered degenerate semiconductors, where the Fermi level lies deeply inside the conduction band in the region of extended states, transport cannot be generally described by the Boltzmann transport theory. In disordered semiconductors, where the Fermi level lies in the region of localized states, a new transport mechanism is possible, related to inelastic tunneling between localized states (hopping). Since localized eigenstates have different energies, transitions between them must be inelastic, and the required energy is usually provided by the interaction with vibrations of the atomic matrix (phonons). Therefore, in contrast to conduction by electrons in extended states, where the interaction with phonons reduces the conductivity, the interaction with phonons stimulates hopping conductivity that vanishes as $T \rightarrow 0 \mathrm{~K}$.

In this chapter we consider some of the existing concepts and approaches to the description of conduction in the region of extended states; hopping processes will be considered in subsequent chapters. The basis of our discussion is the Fermi-liquid approximation, which appeared to be successful in understanding numerous transport properties of strongly disordered solids. We also discuss some important aspects of the effect of electron-electron correlations in the presence of a random potential and their effect on transport properties of an electron gas. The electron-electron interaction effects can appear to be important, especially at low electron concentrations and in this chapter we discuss some of the features of the interplay between disorder and interactions.

\subsection{TRANSPORT BY ELECTRONS IN EXTENDED STATES FAR FROM THE MOBILITY EDGES}

\subsubsection{Weak-scattering theories}

A situation typical of metals corresponds to conduction by a degenerate gas of charge carriers whose energies are close to the Fermi level. Once the disorder is weak, the transport can be described by using the standard Fermi-liquid approach and the Boltzmann theory. In this theory, the quasi-particles (electrons) treated in the nearly free electron approxima- 
tion are assumed to be weakly scattered by disorder (static random potential of structural defects, impurities, etc.). Once the interaction with the random potential is weak and Condition (1.1.1) or (1.1.2) is satisfied, it suffices to keep only the lowest-order terms and to use the Born approximation in the collision integral. The standard transport theory leads to the following expression for the conductivity:

$$
\sigma=\frac{n e^{2}}{m} \tau .
$$

Here $n$ is the concentration of the free charge carriers (quasi-particles), $m$ is the carrier effective mass, and $\tau$ is the transport momentum relaxation time expressed in terms of the scattering cross-section. This expression has a form appearing in the simple Drude theory for the conductivity of a gas of free particles. The theory also makes it possible to calculate other transport coefficients (diffusion coefficient, thermopower, etc.).

For strong structural disorder, transport is not expected to be described by the conventional Boltzmann theory; nevertheless, surprisingly, sometimes the weak scattering approach works quite well for this case. An example is provided by Ziman's theory of liquid metals $[14,15]$, which appeared to be quite successful in describing the properties of many liquid metals. In these systems, the electrons are scattered by the ions of the metal, and one might expect that the electron mean free path is of the order of an ineratomic distance. However, in Ziman's theory, the electrons treated in the nearly free electron approximation are assumed to weakly interact with ions of the disordered matrix of the liquid and their wave functions are taken to be plane waves. Using the expression for the conductivity in terms of the mean free path $l$ from the Boltzmann theory and the measured conductivities, one can estimate $l$ for different liquid metals; it appears that $l$ can be one to two orders of magnitude greater than the interatomic spacing. This indicates that the scattering is indeed weak in spite of substantial disorder. Therefore, one is justified in using the standard transport theory for a weak scattering potential; the scattering is essentially similar to the scattering of X-rays or neutrons by liquids. In this case, the calculation of the conductivity presents no difficulties.

In Ziman's theory, the Born approximation is used; in addition, correlations in the positions of the scattering ions described by the structure factor are taken into account. The conductivity is related to the probability of scattering between the plane wave eigenstates $|\mathbf{k}\rangle$ and $\left|\mathbf{k}^{\prime}\right\rangle$, which is proportional to the square of the matrix element $\left|\left\langle\mathbf{k}|V| \mathbf{k}^{\prime}\right\rangle\right|^{2}$, where $V(\mathbf{x})=\sum_{i} v_{a}\left(\mathbf{x}-\mathbf{R}_{i}\right)$ is the scattering potential equal to the sum of atomic potentials $v_{a}\left(\mathbf{x}-\mathbf{R}_{i}\right)$ of individual centers randomly distributed in space. As for scattering of X-rays or neutrons by liquids, we may write

$$
\left|\left\langle\mathbf{k}|V| \mathbf{k}^{\prime}\right\rangle\right|^{2}=|V(\mathbf{q})|^{2}=N \Omega\left|v_{\mathrm{a}}(\mathbf{q})\right|^{2} S(\mathbf{q}),
$$

where $N$ is the concentration of scattering centers; $\Omega$ is the volume of the system; $V(\mathbf{q})$ and $v_{\mathrm{a}}(\mathbf{q})$ are the Fourier transforms of the total and atomic potentials, respectively; $\mathbf{q}=\mathbf{k}^{\prime}-\mathbf{k}$; $\left|v_{a}(\mathbf{q})\right|^{2}$ is the atomic form factor; $S(\mathbf{q})$ is the structure factor

$$
S(\mathbf{q})=1+\langle\exp (i \mathbf{q} \mathbf{R})\rangle=1+N^{-1} \int \mathrm{d} \mathbf{R} \exp (i \mathbf{q} \mathbf{R}) h(R)
$$


where $\langle\ldots\rangle$ denotes the averaging over impurity configurations and $h(R)$ is the pair correlation function related to the radial distribution function $g(R)$ by the expression $h(R)=g(R)-1$ (here $N g(R) \mathrm{d} \mathbf{R}$ is the probability of finding an atom in the volume element $\mathrm{d} \mathbf{R}$ at a distance $R$ from the origin $\mathbf{R}=0$, provided that there is an atom at the origin). For an isotropic case, we easily obtain the standard expression

$$
S(q)=1+4 \pi N \int \frac{\sin q R}{q R} h(R) R^{2} \mathrm{~d} R .
$$

The standard transport theory leads to Equation (1.2.1) for the conductivity, where $\tau$ is the transport relaxation time, which, for elastic carrier scattering, is given by the expression

$$
\begin{aligned}
\tau_{p}^{-1} & =\frac{2 \pi}{\hbar} \sum_{\mathrm{q}}\left\langle|V(\mathbf{q})|^{2}\right\rangle(1-\cos \theta) \delta\left(\varepsilon_{\mathrm{p}+\mathrm{q}}-\varepsilon_{\mathrm{p}}\right) \\
& =2 \pi\left(\frac{m}{2 \pi \hbar^{2}}\right)^{2} N \Omega v \int_{0}^{\pi} \mathrm{d} \theta \sin \theta(1-\cos \theta)\left|v_{a}(q)\right|^{2} S(q),
\end{aligned}
$$

where $\theta=2 \arcsin (q / 2 k)$ is the scattering angle, and $v_{\mathrm{F}}$ is the electron velocity. For a degenerate electron gas, we have

$$
\tau^{-1}=\frac{8}{\pi}\left(\frac{m}{\hbar^{2}}\right)^{2} N v_{\mathrm{F}} \int_{0}^{1} S\left(2 k_{F} x\right)\left(\Omega v_{a}\left(2 k_{F} x\right)\right)^{2} x^{3} \mathrm{~d} x,
$$

where $v_{\mathrm{F}}$ is the Fermi velocity and $k_{\mathrm{F}}$ is the Fermi momentum.

One of the spectacular applications of Ziman's theory of liquid metals is the interpretation of the temperature dependence of the resistivity of liquid metals. It appears that the resistivity of monovalent metals $(\mathrm{Li}, \mathrm{Na}, \mathrm{K}, \mathrm{Rb}, \mathrm{Cs}$ ) increases with temperature, whereas the resistivity of bivalent metals decreases. The reason for this is clear if one considers the form of the structure factor $S(q)$ that was measured in numerous studies; $S(q)$ is schematically shown in Figure 1.2. The dashed vertical lines correspond to the values $q_{1,2}=2 k_{\mathrm{F}}$ for monovalent and bivalent metals. Since the integrand in Equation (1.2.6) is a rapidly increasing function of $x$, the main contribution to the integral comes from the region near the upper limit, i.e., near the point $q=2 k_{\mathrm{F}}$. With increasing temperature, the correlation in ion positions becomes weaker and $S(q)$ approaches the limiting value $S(q)=1$, corresponding to absolutely random positions of the ions. Thus the value $S\left(q_{1}\right)$ increases with temperature and the value $S\left(q_{2}\right)$ decreases.

The above simplified theory using the Born approximation works quite well for many liquid metals, indicating that the scattering may indeed be considered as weak. There are several reasons for this. First, it is well known in the electronic theory of metals that the nearly free electron model may be used if the actual ion potential is replaced by a smooth pseudopotential incorporating the effect of the core states on the states of free electrons $[16,17]$. Second, the pseudopotential is screened due to electron spatial redistribution. Screening substantially suppresses the pseudopotential compared with the pseudopotential of 'bare' ions [15]. Third, the theory of liquid metals takes into account the correlation in spatial positions of neighboring atoms by introducing the structure factor $S(q)$ (Equation 


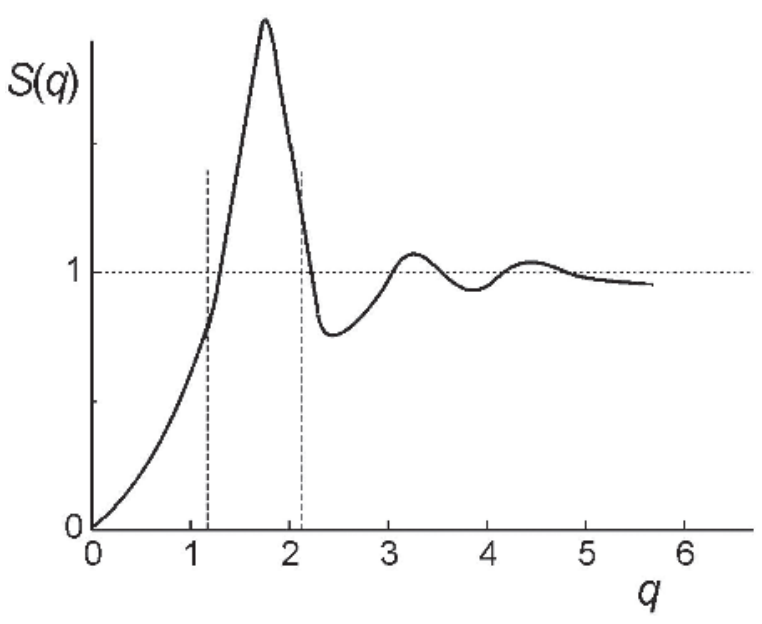

Figure 1.2 Sructure factor $S(q)$ for liquid metals (schematic)

1.2.4). It appears that for many simple metals, the structure factor $S(q)$ is small at small $q$ just in the region where the screened pseudopotential has its maximum, whereas for the values of $q$, where the structure factor is large, the pseudopotential is small.

A weak-scattering theory may be also applied to degenerate heavily doped semiconductors, where the Fermi level lies in the region of extended states not very close to the mobility edge. The condition for applicability of this theory is $n a_{\mathrm{B}}^{3}>1$, where $n$ is the electron concentration, assumed to be equal to the concentration of the doping impurity, and $a_{\mathrm{B}}$ is the Bohr radius; it appeared to be possible to construct an asymptotic theory for the electron energy spectrum and electron transport [18]. Once the impurity concentration is not too high (the average interimpurity distance is much greater than the lattice period), one may consider Bloch electrons scattered by screened atomic impurity potentials; moreover, the impurity atoms may be considered as randomly distributed in space. Due to screening, the scattering appears to be weak in the asymptotic limit $n a_{\mathrm{B}}^{3} \gg>1$ and the Born approximation may be used giving Equation (1.2.1). A straightforward calculation of the higher-order terms describing both the terms corresponding to higher Born approximations for scattering by an impurity atom and to correlated many-impurity scattering were analyzed, thus establishing the applicability conditions for the transport theory in question $[19,20]$. The result of the perturbation theory for transport coefficients of a degenerate semiconductor with screened ionized impurities is that the perturbation series is expected to be slowly converging and lowest-order corrections to the conductivity are of the form [19]

$$
0.3 \ln ^{-1}\left(n a_{\mathrm{B}}^{3}\right)+0.3\left(n a_{\mathrm{B}}^{3}\right)^{-1 / 6} .
$$

These corrections obtained for $n a_{\mathrm{B}}^{3}>>1$ decrease very slowly with increasing $n a_{\mathrm{B}}^{3}$. Thus using a weak scattering theory, one can expect to obtain only qualitative agreement with experiment $[9,21]$. In addition, for germanium and silicon, one has to take into account the multi-valley structure of the conduction band [22].

Another example of a disordered system, for which a reliable theory exists for charge carrier transport via extended states above the mobility edge, is provided by semiconductor 
solid solutions with short-range random disorder potential of the white-noise type [23-26]. Semiconductor solid solutions $\mathrm{A}_{x} \mathrm{~B}_{1-x}$ or, in other words, mixed crystals, are crystalline semiconductors in which sites of the crystalline lattice can be occupied by atoms of different types $\mathrm{A}$ and $\mathrm{B}$; here $x(0 \leq x \leq 1)$ is the probability that a site is occupied by an A atom. Due to the random spatial distribution of $\mathrm{A}$ and $\mathrm{B}$ atoms, local statistical fluctuations of the composition $x$ along the sample are unavoidable. Since the position of the band edge depends on composition, the fluctuations of the band edge appear, similar to those produced by electrostatic disorder potential. To be specific, we consider the effect of the random potential on conduction band electrons. Let $\varepsilon_{\mathrm{c}}(x)$ be the conduction band minimum for a crystal with composition $x$. In Figure 1.3, a possible dependence $\varepsilon_{\mathrm{c}}(x)$ is shown schematically. If the average composition for the whole sample is $x_{0}$, the local positions of the band edge $\varepsilon_{\mathrm{c}}(x)$ fluctuate about the average value $\varepsilon_{\mathrm{c}}\left(x_{0}\right)$, according to the fluctuations of the composition $x$ about $x_{0}$. At small deviations $\Delta x$ of the composition from the average value, one can use the linear relation

$$
\varepsilon_{\mathrm{c}}\left(x_{0}+\Delta x\right)=\varepsilon_{\mathrm{c}}\left(x_{0}\right)+\alpha \Delta x
$$

where

$$
\alpha=\left.\frac{\mathrm{d} \varepsilon_{\mathrm{c}}(x)}{\mathrm{d} x}\right|_{x=x_{0}} .
$$

If the deviation of the concentration of $\mathrm{A}$ atoms from its mean value in some region of a sample is $\xi(\mathbf{r})$ and the total concentration of lattice sites is $N$, the deviation of the composition in this region is $\Delta x=\xi(\mathbf{r}) / N$ and the potential energy of an electron at the bottom of the conduction band is

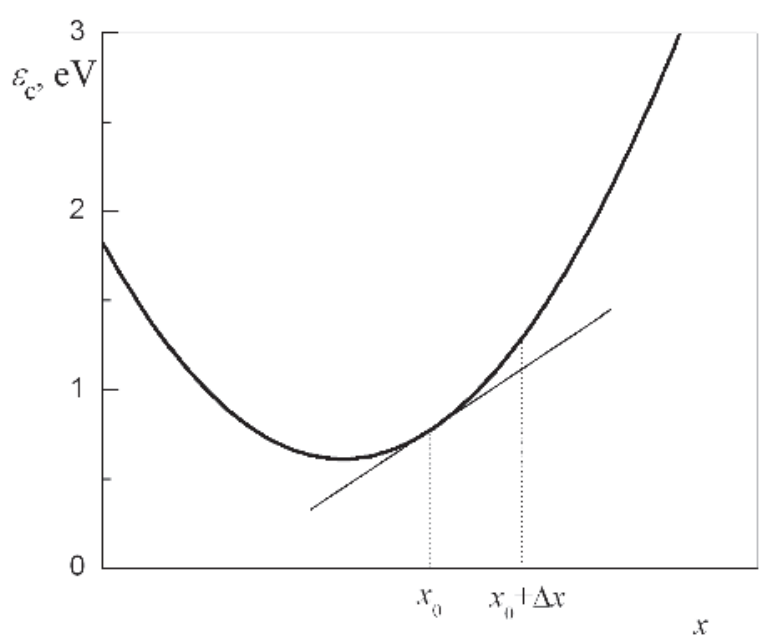

Figure 1.3 Composition dependence of the conduction band edge $\varepsilon_{\mathrm{c}}(x)$ in a mixed crystal (schematic) 


$$
V(r)=\alpha \frac{\xi(\mathbf{r})}{N}
$$

Although the disorder in such systems is called a 'short-range' disorder, it should be realized that this description is valid only for size fluctuations much larger than the lattice constant of the material. The term 'short-range' is due to the assumption on the absolutely uncorrelated statistical properties of the disorder. This means that potential amplitudes at adjacent spatial points are completely uncorrelated. Indeed, it is usually assumed that the correlation function of disorder in mixed crystals can be approximated by a white-noise correlation function of the form

$$
\left\langle\xi(\mathbf{r}) \xi\left(\mathbf{r}^{\prime}\right)\right\rangle=x(1-x) N \delta\left(\mathbf{r}-\mathbf{r}^{\prime}\right) .
$$

Then the random potential created by such composition fluctuations is described by the correlation function [26]

$$
\left\langle V(\mathbf{r}) V\left(\mathbf{r}^{\prime}\right)\right\rangle=\gamma \delta\left(\mathbf{r}-\mathbf{r}^{\prime}\right)
$$

where

$$
\gamma=\frac{\alpha^{2}}{N} x(1-x)
$$

Charge carriers in mixed crystals are scattered by composition fluctuations. As usual in the kinetic description of free electrons, the fluctuations with a space scale of the order of the electron wavelength are most efficient in scattering. We have

$$
\left\langle\left|V_{\mathrm{q}}\right|^{2}\right\rangle=\frac{1}{\Omega} \int \mathrm{dr} \exp (i \mathbf{q r})\langle V(\mathbf{r}) V(0)\rangle
$$

and using the correlation function (Equation 1.2.12), we obtain the relation

$$
\left\langle\left|V_{\mathrm{q}}\right|^{2}\right\rangle=\frac{\alpha^{2} x(1-x)}{\Omega N},
$$

which shows that the scattering by composition fluctuations is equivalent to that by a shortrange potential [25]. Substituting Equation (1.2.15) into Equation (1.2.5), we find [26]

$$
\tau_{\mathrm{p}}^{-1}=\frac{\alpha^{2} x(1-x) m k}{\pi \hbar^{3} N} .
$$

This formula leads to the electron mobility of the form $[25,26]$

$$
\mu_{\mathrm{C}}=\frac{\pi^{3 / 2}}{2 \sqrt{2}} \frac{e \hbar^{4} N}{\alpha^{2} x(1-x) m^{5 / 2}(k T)^{1 / 2}} .
$$


Very similar formulas can be found in many recent publications (see, for example [27]). It has also been modified for applications to two-dimensional systems [28] and to disordered dilute magnetic semiconductors [29].

This theoretical description can be applied to other disordered systems, provided that the correlation function of the disorder potential has the form of Equation (1.2.12) with a known amplitude $\gamma$. However, it should be noted that this short-range disorder of a whitenoise type is only a simple model, which can hardly be justified for most disordered materials.

The Boltzmann theory predicts that, in the low-temperature region, charge carrier scattering is dominated by random static potential (to be specific, that of impurities). This scattering is elastic, its probability remains finite at $T=0$, and the conductivity is almost independent of temperature. Some temperature dependence of the conductivity may exist due to the temperature dependence of the scattering processes. For most scattering mechanisms, it follows from the classical Bolzmann equation that scattering probability and, hence, the resistivity increase with temperature, i.e., the temperature coefficient of the resistivity is positive: $\mathrm{d} \rho / \mathrm{d} T>0$. This is usually called a normal metallic behavior of the resistivity.

The temperature dependence of the conductivity may be due to phonon scattering whose probability increases with increasing temperature, giving rise to a positive temperature coefficient, predominantly due the increase in the number of phonons. At high temperatures, it is proportional to $k T /(\hbar \omega)$ (where $\omega$ is the phonon frequency); accordingly, we have $\sigma \sim T^{-1}$. At low temperatures, the temperature dependence of the conductivity is stronger (the Bloch-Grüneisen law, $\sigma \sim T^{-5}$ ).

Scattering by static disorder (impurities and defects) may also give rise to some temperature dependence of the conductivity. This is related mainly to screening effects. Screening becomes weaker with increasing temperature so that, for this scattering mechanism, one can also expect a positive temperature coefficient of the resistivity, i.e., normal metallic behavior.

Yet another scattering mechanism is electron-electron interaction. Since for a degenerate electron gas, only the electrons in the layer of width of the order of $k T$ near the Fermi level can be scattered, the scattering probability is proportional to $T^{2}$. The electron-electron collisions are substantially inelastic, the energy variation being of the order of $k T$. Thus classical electron-electron scattering is again expected to result in normal metallic behavior of a degenerate electron gas at low temperatures.

However, in the low-temperature range, the temperature dependence of the classical conductivity described by the Boltzmann theory becomes weaker and the effects related to quantum interference of scattered electron waves can become important. These effects that lie beyond the classical approach are discussed in Section 1.2.2.

\subsubsection{Weak localization}

An important feature of scattering by static disorder is that the elastic scattering is coherent and, therefore, when considering higher-order terms in the calculation of the conductivity, one must take into account the interference of the electron waves scattered by different impurities. This interference gives rise to quantum interference corrections to the Drude conductivity producing a weak localization of the electronic states [30]. Inelastic effects 
such as scattering by phonons and by other electrons destroy the phase coherence, and the weak localization, being an interference phenomenon, can be suppressed by inelastic collisions. An explicit form of the interference corrections to the conductivity obtained by summing the singular backscattering terms of the perturbation expansion in powers of the impurity concentration are [30]

$$
\begin{gathered}
\Delta \sigma_{3 \mathrm{D}}=-\left(\frac{e^{2}}{\pi^{3} \hbar}\right)\left(\frac{1}{l}-\frac{1}{L_{\varphi}}\right), \\
\Delta \sigma_{2 \mathrm{D}}=-\left(\frac{e^{2}}{\pi^{2} \hbar}\right) \ln \left(\frac{L_{\varphi}}{l}\right)
\end{gathered}
$$

for three- and two-dimensional systems, respectively (we do not discuss one-dimensional systems here). Here $L_{\varphi}=\sqrt{D \tau_{\varphi}}, D$ is the diffusion coefficient determined by the impurity elastic scattering, $\tau_{\varphi}$ is the phase-breaking time, and $l$ is the elastic mean free path. In Equations $(1.2 .18,1.2 .19), L_{\varphi}$ and $l$ appear as natural cutoffs for the size of closed electron paths when summing over all closed paths corresponding to coherent electron propagation. It should be noted that the phase-breaking time $\tau_{\varphi}$ does not always coincide with the inelastic collision time $\tau_{\text {in }}[31,32]$. Indeed, if the energy change $\Delta \varepsilon$ in an individual collision is small compared with $\hbar / \tau_{\text {in }}$, the phase variation in a single scattering event is about $\Delta \varepsilon \tau_{\text {in }} / \hbar$ and may be small compared with $2 \pi$. This may be the case for electron-electron scattering. For phonon scattering, the phase variation is usually not small, and $L_{\varphi} \simeq l_{\text {in }}$, where $l_{\text {in }}$ is the inelastic mean free path.

As discussed in Section 1.2.1, the inelastic scattering time depends on temperature, increasing as temperature decreases. Let $\tau_{\text {in }} \sim T^{-p}$ where $p$ is an index depending on the scattering mechanism, dimensionality, etc. Using $l_{\text {in }}=a T^{-p / 2}$ in place of the cutoff length $L_{\varphi}$ in corrections (Equations 1.2.18, 1.2.19) to the conductivity, we obtain

$$
\begin{gathered}
\Delta \sigma_{3 \mathrm{D}}(T)=-\left(\frac{e^{2}}{\pi^{3} \hbar}\right)\left(\frac{1}{l}-\frac{1}{a} T^{p / 2}\right), \\
\Delta \sigma_{2 \mathrm{D}}=\frac{p e^{2}}{2 \pi^{2} \hbar} \ln \left(\frac{T}{T_{0}}\right),
\end{gathered}
$$

where $T_{0}=(a / l)^{2 / p}$. In the low-temperature range where the Drude conductivity is independent of temperature, the temperature dependence of the quantum interference corrections (Equations 1.2.20, 1.2.21) is important, although corrections must be small in the region of applicability of the perturbation theory. This temperature dependence corresponds to the increase in conductivity with $T$, similar to that typical of localized state conduction.

Other phase-breaking mechanisms can also affect the conductivity, in particular, related to the dephasing effect of a magnetic field, magnetic impurity, and spin-orbit scattering. A magnetic field $B$ destroys time-reversal symmetry and provides a magnetic length cutoff $L_{H}=(e B / \hbar)^{1 / 2}[33]$. Indeed, the magnetic field introduces phase shifts for electrons traveling along a closed path in opposite directions. Since one must average over all possible paths, the average interference correction vanishes for paths of size exceeding $L_{H}$, i.e., magnetic field suppresses the localization effect. It turns out that the magnetoresistance due to this effect is always negative. Furthermore, since $L_{\varphi}$ can be quite large, the characteristic 
magnetic field can be very small, of the order of several mT. According to Equation (1.2.19), in two dimensions, the magnetoresistance varies logarithmically with $B$. Lee [34] has shown that magnetic impurities destroy the coherence, so that on a length scale greater than $L_{\mathrm{s}}=\sqrt{D \tau_{\mathrm{s}}}$, where $\tau_{\mathrm{s}}$ is the spin-flip time, the conductivity is no longer dependent on length. In the case of spin-orbit coupling, the quantum correction to the classical (Drude) conductivity is positive and the effect of the spin-orbit interaction is sometimes called weak anti-localization [35].

It should be noted that for low-mobility samples and at low temperatures, which are needed for the observation of weak-localization corrections so that condition $L_{\varphi} \gg l$ can be satisfied, the cutoff length can be greater than the sample size $L$. In this case, the quantum correction to the conductivity given by (Equations $1.2 .18,1.2 .19$ ), where $L_{\varphi}$ is replaced by $L$, does not depend on temperature.

\subsubsection{Interaction effects}

The Fermi-liquid theory is currently used to describe the effect of the electron-electron interaction on the electronic properties of pure metals. For nonideal 'dirty' metals, the role of interaction effects can be substantially different from the case of almost free quasiparticles. Apart from the weak-localization effects, elastic scattering by impurities in metals can substantially modify the electron-electron interaction. Let the elastic momentum relaxation time $\tau$ be small compared with the characteristic time $\hbar /(k T)$ required for two interacting quasi-particles to change their energy by a value of about $k T$, i.e., let

$$
k T \tau / \hbar<<1
$$

This means that two interacting particles diffuse coherently (are scattered many times by impurities) before they exchange an energy of about $k T$; this electron-electron interaction regime is 'diffusive'. In the diffusive regime, the electrons spend a longer time in a given region of space compared with the plane-wave states, and their interaction is enhanced.

Under Condition (1.2.22), the motion of electrons during the characteristic time of electron-electron collisions is characterized by the diffusion coefficient $D=v_{\mathrm{F}}^{2} \tau / 3$, where $v_{\mathrm{F}}$ is the Fermi velocity. The electron-electron interaction produces a cusp in the electronic density of states at the Fermi level $[36,37]$. The physical reason for such a variation in the density of extended states near the Fermi level is related to the shift of the energy of a particle added into the system due to the Coulomb interaction with electrons of the occupied states. Thus, the nature of the cusp is similar to that of the Coulomb gap appearing at the Fermi level in the region of localized states [5]. This feature produces the anomaly in the tunneling current at zero bias which is often observed when studying the current-voltage characteristics of tunneling contacts (see, e.g., [38-40]).

It has long been recognized that, in addition to the weak-localization correction, the interaction between the electrons that coherently diffuse due to impurity scattering also gives rise to the quantum correction to the conductivity, which has the form $[8,33,36]$

$$
\Delta \sigma_{3 \mathrm{D}}^{(\mathrm{int})}(T)=A \frac{e^{2}}{\hbar}\left(1-\frac{9}{8} F_{0}^{\alpha}\right)\left(\frac{k_{\mathrm{B}} T}{\hbar D}\right)^{1 / 2},
$$




$$
\Delta \sigma_{2 \mathrm{D}}^{(\mathrm{int})}=\frac{e^{2}}{2 \pi^{2} \hbar}\left\{1+3\left(1-\frac{\ln \left(1+F_{0}^{\alpha}\right)}{F_{0}^{\alpha}}\right)\right\} \ln (k T \tau / \hbar)
$$

in three-dimensional and two-dimensional cases, respectively. Here $A$ is a constant ( $A=1.3 / 3 \sqrt{2} \pi^{2},[8]$ ) and the constants $F_{0}^{\alpha}$ are the Fermi-liquid interaction parameters (in the triplet channel) that depend on the screening radius. Thus the interaction term has a $T^{1 / 2}$ dependence, but its sign depends on the relative size of the exchange and Hartree terms, which is a function of the screening length. In doped multi-valley semiconductors, such effects as the presence of several conduction band minima, scattering, and mass anisotropy must be considered if a detailed quantitative comparison is desired.

We see that the temperature dependence of the conductivity is similar for localization and interaction corrections, both for the three-dimensional and two-dimensional cases. However, it is possible to distinguish between the corresponding contributions experimentally, using the measurements in a magnetic field. Localization corrections are suppressed by a magnetic field, producing a negative magnetoresistance, whereas the interaction magnetoresistance is positive (isotropic for spin splitting and transverse for the orbital part). The Hall coefficient $R_{\mathrm{H}}$ is another quantity that behaves differently for localization and interaction effects. In particular, there is no weak-localization correction to the Hall coefficient [41], whereas in the interaction theory $\delta R_{\mathrm{H}} / R_{\mathrm{H}}=2 \delta \rho / \rho$, where $\delta \rho$ is the logarithmic correction to the resistivity $\rho$.

The regime corresponding to the condition

$$
k T \tau / \hbar>>1
$$

is called 'ballistic'; in this regime, the time of the energy exchange is much shorter than $\tau$ and the electron-electron interaction is mediated by a single impurity. The Friedel electron density oscillations appear around an impurity with a short-range scattering potential so that an electron is backscattered from the impurity as well as from the Friedel oscillations. Constructive interference of the two scattered waves, which gives rise to a linear correction to the Drude conductivity, dependent on the Fermi-liquid interaction constant in the triplet channel $F_{0}^{\alpha}$, in two-dimensions is [42]

$$
\delta \sigma(T)=\sigma(0)\left(1+\frac{3 F_{0}^{\alpha}}{1+F_{0}^{\alpha}}\right) \frac{k T}{\varepsilon_{\mathrm{F}}} .
$$

The expression in the brackets in Equation (1.2.26) consists of two contributions: one of the exchange processes and the second of the Hartree interaction. The sign of the temperature dependence of the conductance is determined by the sign and magnitude of the interaction parameter $F_{0}^{\alpha}$. If $F_{0}^{\alpha}$ is sufficiently large and negative, we have $\mathrm{d} \delta \sigma / \mathrm{d} T<0$ and this corresponds to metallic-type conduction.

Experimental studies of the corrections to the Drude conductivity has shown that in most cases, interaction corrections cannot be disregarded in both three-dimensional and twodimensional systems [8]. This is not unexpected at low concentrations, where the role of Coulomb interaction is known to become important. The role of interaction may be characterized by the dimensionless Wigner-Seitz parameter $r_{\mathrm{s}}$, defined as the average 
dimensionless distance between charge carriers measured in units of the effective Bohr radii $a_{\mathrm{B}}=4 \pi \kappa_{0} \kappa \hbar^{2} /\left(m e^{2}\right)$, where $\kappa$ is the permittivity and $m$ is the effective mass. For a three-dimensional degenerate electron gas, we have $r_{\mathrm{s}}^{(3 \mathrm{D})}=(3 / 4 \pi)^{1 / 3}\left(n^{(3 \mathrm{D})} a_{\mathrm{B}}^{3}\right)^{-1 / 3}$ and for two-dimensional systems, $r_{\mathrm{s}}^{(2 \mathrm{D})}=\pi^{-1 / 2}\left(n^{(2 \mathrm{D})} a_{\mathrm{B}}^{2}\right)^{-1 / 2}$. The ratio $U_{\mathrm{C}} / \varepsilon_{\mathrm{F}}$ of the Coulomb energy of electrons at an average distance to the Fermi energy is expressed in terms of the parameter $r_{\mathrm{s}}$. Thus, for a degenerate three-dimensional electron gas, we have $U_{\mathrm{C}} \simeq \varepsilon_{\mathrm{B}} / r_{\mathrm{s}}^{(3 \mathrm{D})}$, $E_{\mathrm{F}}=(9 \pi / 4)^{2 / 3} \varepsilon_{\mathrm{B}}\left(r_{\mathrm{s}}^{(3 \mathrm{D})}\right)^{-2 / 3}$, and $U_{\mathrm{C}} / \varepsilon_{\mathrm{F}} \simeq(4 / 9 \pi)^{2 / 3}\left(r_{\mathrm{s}}^{(3 \mathrm{D})}\right)^{-1 / 3}$; here, $\varepsilon_{\mathrm{B}}=e^{2} /\left(2 \kappa a_{\mathrm{B}}\right)$ is the effective Bohr energy. For some semiconductor structures (e.g., silicon-based), one must additionally take into account the valley degeneracy. For the two-dimensional case, we have $U_{\mathrm{C}} \simeq \varepsilon_{\mathrm{B}} / r_{\mathrm{s}}^{(2 \mathrm{D})}, \varepsilon_{\mathrm{F}}=\varepsilon_{\mathrm{B}}\left(r_{\mathrm{S}}^{(2 \mathrm{D})}\right)^{-2}$, and $U_{\mathrm{C}} / \varepsilon_{\mathrm{F}} \simeq r_{\mathrm{s}}^{(3 \mathrm{D})}$. In both three-dimensional and twodimensional systems, the interaction effects are seen to be important at large $r_{\mathrm{s}}$, i.e., at low electron concentrations, in particular, near the metal-insulator transition. For semiconductor structures typically studied, the values of $r_{\mathrm{s}}$ are often quite large (thus, for GaAs/AlGaAs heterostructures, the values of $r_{\mathrm{s}}$ are in the range 10-17 for a two-dimensional hole gas and 1-2.5 for a two-dimensional electron gas [42]).

Since the experiments clearly indicate the presence of weak-localization corrections, electron-electron interaction effects cannot be generally disregarded, in particular, in the energy region near the mobility edge. Nevertheless, the concept of noninteracting charge carries has appeared to be very fruitful for understanding the main features of extendedstate conduction, including the vicinity of the metal-insulator transition. A basis for the description of transport by noninteracting charge carriers is provided by the one-parameter scaling theory of localization considered in Section 1.3.

\subsection{SCALING THEORY OF LOCALIZATION}

\subsubsection{Main ideas of the scaling theory of localization}

Transition from the region $\varepsilon_{\mathrm{F}}>\varepsilon_{\mathrm{c}}$ to the region $\varepsilon_{\mathrm{F}}<\varepsilon_{\mathrm{c}}$ is in a sense similar to a phase transition, and the difficulties arising in the description of the properties of the system in the vicinity of the localization threshold are similar to those in the theory of thermodynamic phase transitions. On the other hand, the analogy with phase transitions suggests the possibility of applying some methods of the modern theory of critical phenomena to the problem of Anderson localization. One of them is the method of the renormalization group, including scaling transformations. The scaling theory of the localization suggested by Abrahams et al. [43] is based on certain assumptions on the behavior of the conductance $G$ of the system under scaling transformations. The conductance $G$ is expressed in terms of the conductivity by

$$
G=\sigma L^{d-2}
$$

where $L$ is the linear size of the system chosen in the form of a hypercube and $d$ is its dimensionality.

The conductance of the system $G$ is directly related to the localization of electronic wavefunctions. According to Thouless [44], the degree of localization of wavefunctions in the bulk of the system is related to the sensitivity of energy levels to the variation in boundary conditions, and the shift of the levels with varying boundary conditions is expressed in terms of the conductance. Let $\zeta$ be the shift of an energy level if the boundary conditions 
are changed, e.g., from periodic to antiperiodic. Then for the states localized in the bulk, the quantity $\zeta$ is exponentially small, provided that the localization radius $\alpha$ is smaller than the system size $L, \zeta \sim \exp (-L / \alpha)$. The effect of boundary conditions on extended states can be estimated using the following argument [45]. For $L \gg l$, where $l$ is the mean free path with respect to elastic collisions, the electron has time to be multiply scattered before it reaches the boundary of the system. The motion of an electron undergoing frequent elastic coherent collisions (without breaking the phase of the wave function) has a diffusive character. Let $D$ be the corresponding diffusion coefficient; then the effect of the boundaries on the electron motion becomes important in time $t \sim L^{2} / D$ when the electron reaches the boundary. On the other hand, one can estimate the time $t$ after which the effect of boundary conditions becomes important in a different way. This is the time $t$ after which the variation $\zeta t / \hbar$ in the phase of the wave electron function produced by a change in the boundary conditions becomes of the order of unity, i.e., $t \sim \hbar / \zeta$. Comparing the two expressions for the time $t$, we obtain

$$
\zeta L^{2} / D \hbar \sim 1
$$

It turns out that the quantity $\zeta$ is related to the diffusion coefficient, and by Einstein's relation, to the conductivity. For degenerate $d$-dimensional electron gas, we may write Einstein's relation as

$$
\sigma=e^{2} D \rho_{\mathrm{F}}=e^{2} D L^{-d} w^{-1}
$$

where $n$ is the concentration of charge carriers, $\rho_{\mathrm{F}}$ is the density of states at the Fermi level, and $w=\left(\rho_{\mathrm{F}} L^{d}\right)^{-1}$ is the characteristic interlevel separation. Then we obtain from Equation (1.3.2)

$$
G=\sigma L^{d-2}=\left(e^{2} / \hbar\right) \zeta / w
$$

Accordingly, the dimensionless conductance

$$
g=G \hbar / e^{2}=\zeta / w
$$

is directly proportional to the shift of the energy level at the change in boundary conditions and characterizes the degree of localization of electronic wave functions.

From Equation (1.3.3), we see that $d=2$ is the critical dimensionality for the problem in question, and the localization properties of electronic states in systems with $d<2$ and $d>2$ are different. Indeed, for a system of size $L>l$ and extended electron states, one can define conductivity, independent of $L$. According to Equation (1.3.3), for $d<2$, the relative level shift decreases with increasing $L$, i.e., the tendency to localization is realized, whereas for $d>2$, the quantity $\zeta / w$ increases with the system size.

\subsubsection{The main equations of one-parameter scaling}

A qualitative analysis of the behavior of the function $g(L)$, i.e., of the localization properties, can be performed using the assumption of scaling invariance. To this aim, one can perform a scaling transformation similar to Kadanoff's transformation in the theory of 
phase transitions [46]. Consider a large hypercube (with an edge $L>l$ ) of volume $L^{d}$ in the $d$-dimensional space, where the function $g(L)$ is defined by Equations (1.3.3) and (1.3.4), and then pass to a large system of linear size $b L$ constructed from $b^{d}$ such hypercubes. Assuming that the shift of energy levels when constructing the large system of size $b L$ from the original hypercubes is determined only by the quantity $g(L)$, one can write

$$
g(b L)=f[b, g(L)]
$$

This is a basic assumption of the scaling theory of localization [43], related to the assumption that there is a unique scale (of length or energy) that determines the behavior of physical parameters in the critical region (one-parameter scaling).

Equation (1.3.5) can be written in a differential form similar to the Gell-Mann-Low equation in quantum electrodynamics. To this aim, we assume that the parameter $b$ in Equation (1.3.5) varies continuously and differentiate with respect to $b$, setting then $b=1$. Thus we find

$$
\frac{\mathrm{d} \ln g(L)}{\mathrm{d} \ln L}=\beta[g(L)]
$$

where

$$
\beta[g(L)]=g^{-1}[\partial f(b, g) / \partial b]_{b=1}
$$

is the scaling function similar to the Gell-Mann-Low function. The differential form (Equation 1.3.6) often appears to be more convenient for the analysis of the conductance $g(L)$ than the scaling relation (Equation 1.3.5).

The function $\beta(g)$, which appears in Equation (1.3.6), depends only on the dimensionality of the system. Its asymptotic form at small and large $g$ can be found by the following arguments. If $g$ is small, i.e., $\zeta / w$ is small, then the states are localized, and in a space of any dimensionality we have $g(L) \sim g_{1} \exp (-L / \alpha)$ at large $L$. From this, we obtain

$$
\beta(g) \rightarrow \ln \left(g / g_{1}\right) \quad \text { as } g \rightarrow 0 .
$$

On the contrary, for large $\beta(g)$, macroscopic theory of transport phenomena may be used. The asymptotic form of the function $\beta(g)$ at large $g$ can be found taking account of the quantum interference corrections to the result of the transport theory based on the Boltzmann equation (see Section 1.2). For the three-dimensional case, Equation (1.2.18) gives, for the size-dependent correction to the conductivity, $g(L)=L\left(g_{0}+A / L\right)$, where $g_{0}$ is the conductance of the infinitely large system and $A=\pi^{-3}$. From this, we easily obtain that at large $g$

$$
\beta(g) \simeq 1-A / g
$$

For a two-dimensional metal, the asymptotic form of the function $\beta(g)$ at large $g$ is determined by the correction (Equation 1.2.19) to the transport equation, related to the backscattering, whose sum logarithmically diverges at small wave vectors $k$. Using Equation (1.2.19), we obtain 


$$
\beta(g) \simeq-A / g,
$$

at large $g$. Combining the results for systems of different dimensionalities, at large $g$ we obtain

$$
\beta(g) \rightarrow d-2-A / g
$$

where $d$ is the system dimensionality and $A$ is, generally, different for $d=2$ and $d=3$. Using asymptotic expressions (1.3.7) and (1.3.10) and assuming that the function $\beta(g)$ is continuous and monotonic, we can qualitatively represent its shape in the entire range of variation of $g$. For systems of different dimensionalities, the function $\beta(g)$ is schematically plotted in Figure 1.4.

In Figure 1.4, we see that, for $d=3$ (curve $a$ ), there is at least one zero of the function $\beta(g)$, which we denote by $g_{\mathrm{c}}$. This zero corresponds to an unstable fixed point of Equation (1.3.6). Indeed, let a system of size $L_{0}$ have a conductance $g\left(L_{0}\right) \equiv g_{0}>g_{\text {c }}$ and let the system size $L>L_{0}$ be monotonically increased. Since $\beta(g)>0$ for $g>g_{c}$, the conductance $g$ increases with $L$ and the function $\beta(g)$ also increases, moving away from the point $g_{c}$ and asymptotically approaching the value $\beta=1$ at large $g$. This means that, for large systems, the conductance is proportional to $L$, i.e., the conductivity tends to a constant value $\sigma_{0}$ as $L \rightarrow \infty$, as expected for extended states. On the contrary, if initially the conductance $g_{0}$ is smaller than $g_{c}$, then we obtain that the conductivity exponentially decreases as $L \rightarrow \infty$, i.e., it corresponds to localized states. Thus, the fixed point $g_{\mathrm{c}}$ separates the regions of the initial conditions corresponding to localized and extended states. The quantity $\left(g_{0}-g_{\mathrm{c}}\right) / g_{\mathrm{c}}$ may be considered as a parameter that determines the energy separation from the mobility edge.

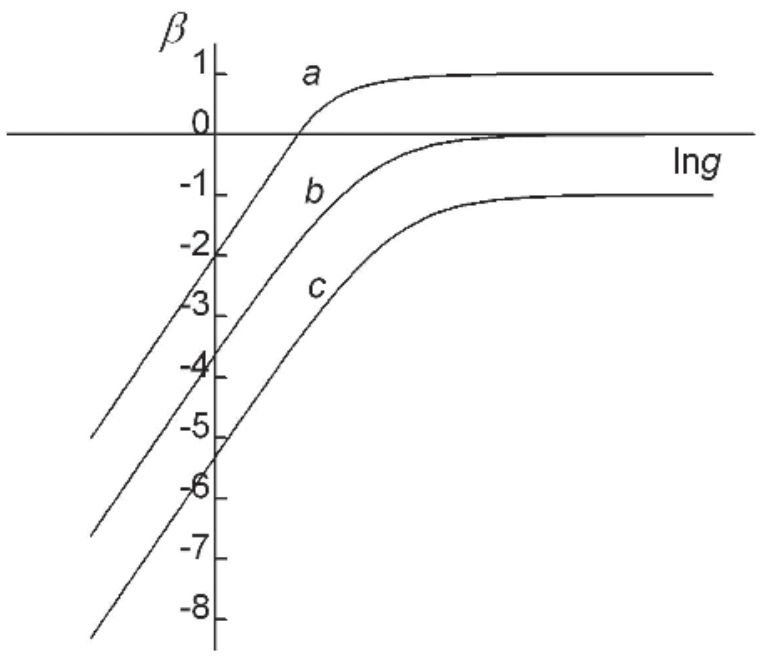

Figure 1.4 The shape of the scaling function $\beta(\mathrm{g})$ for systems of different dimensionalities: (a) $d=3$; (b) $d=2$; (c) $d=1$ 


\subsubsection{Model solutions}

Since, by assumption, the function $\beta(g)$ is smooth, near the fixed point $g_{c}$ it may be approximated by

$$
\beta(g)=\left(g-g_{\mathrm{c}}\right) /\left(v g_{\mathrm{c}}\right),
$$

where the parameter $v$ determines the slope of the function $\beta(g)$ at the point $g=g_{\mathrm{c}}$. However, increasing $L$, we inevitably leave the vicinity of the point $g_{\text {c }}$ where the linear approximation (Equation 1.3.11) is valid. For a qualitative discussion, we may use the model interpolation expression

$$
\beta(g)=\frac{\left(g-g_{\mathrm{c}}\right)}{g-(1-v) g_{\mathrm{c}}},
$$

which gives the correct slope of $\beta(g)$ at $g=g_{\mathrm{c}}$ and the correct asymptotic behavior at large $L$ (if $v g_{\mathrm{c}}=A$ ). Using this expression, we can explicitly perform the integration and find the macroscopic conductivity corresponding to a given initial value $g_{0}$. The final result for the conductivity is only slightly affected by the detailed behavior of the function $\beta(g)$ in the region of intermediate $g$.

Equation (1.3.6) with the function $\beta(g)$, defined by Equation (1.3.12), is easily integrated and we obtain

$$
\left(\frac{g-g_{\mathrm{c}}}{g_{\mathrm{M}}-g_{\mathrm{c}}}\right)^{v}\left(\frac{g}{g_{\mathrm{M}}}\right)^{1-v}=\frac{L}{l} .
$$

Here, the 'initial' (in $L$ ) condition was used corresponding to the lower 'cutoff' at the microscopic length by setting $g(l)=g_{\mathrm{M}}>g_{\mathrm{c}}$. From Equation (1.3.13), we find for the conductivity at large $L$ (for $g \gg g_{c}$ )

$$
\sigma=\frac{g}{L} \simeq \frac{e^{2}}{\hbar l}\left(\frac{g_{\mathrm{M}}-g_{\mathrm{c}}}{g_{\mathrm{c}}}\right)^{v}\left(1+\frac{v g_{\mathrm{c}}}{g}\right)=\frac{e^{2}}{\hbar}\left(\frac{1}{\xi}+\frac{v g_{\mathrm{c}}}{L}\right),
$$

where we have introduced the correlation length

$$
\xi=l\left(\frac{g_{\mathrm{M}}-g_{\mathrm{c}}}{g_{\mathrm{c}}}\right)^{-v}
$$

(here $\left.\left(g_{\mathrm{M}}-g_{\mathrm{c}}\right) / g_{\mathrm{c}}<<1\right)$. It follows from Equation (1.3.13) that, for a system of size $L>>$, $g$ depends linearly on $L$ (this is sometimes called the Ohmic regime), and the conductivity in the limit $L \rightarrow \infty$ is

$$
\sigma=\frac{e^{2}}{\hbar \xi}
$$

the dependence of $g$ on $L$ is nonlinear for finite $L<\xi$. 
Since charge transport is due to electrons with the Fermi energy $\varepsilon_{\mathrm{F}}, g_{\mathrm{M}}$ is the conductance at this energy. By assumption, the quantity $g_{\mathrm{M}}$ is a smooth function of the Fermi energy $\varepsilon_{\mathrm{F}}$ and the conductivity vanishes for $g_{\mathrm{M}}=g_{\mathrm{c}}$ corresponding to the mobility edge $\varepsilon_{\mathrm{c}}$. Expanding $g_{\mathrm{M}}\left(\varepsilon_{\mathrm{F}}\right)$ near $\varepsilon_{\mathrm{c}}$, we obtain

$$
g_{\mathrm{M}}\left(\varepsilon_{F}\right)=g_{\mathrm{c}}+\mathrm{d} g_{\mathrm{M}}\left(\varepsilon_{F}\right) /\left.\mathrm{d} \varepsilon_{\mathrm{F}}\right|_{\varepsilon_{\mathrm{F}}=\varepsilon_{\mathrm{c}}}\left(\varepsilon_{\mathrm{F}}-\varepsilon_{\mathrm{c}}\right) .
$$

Thus, according to Equation (1.3.15), $v$ describes the divergence of the correlation length and the continuous decrease in the conductivity at $T=0 \mathrm{~K}(1.3 .16)$ as the Fermi level $\varepsilon_{\mathrm{F}}$ approaches the mobility edge $\varepsilon_{\mathrm{c}}$. Using Equation (1.3.17), we obtain

$$
\sigma=\frac{e^{2}}{\hbar l}\left(\frac{\varepsilon_{\mathrm{F}}-\varepsilon_{\mathrm{c}}}{\varepsilon^{*}}\right)^{v}
$$

where we have introduced the characteristic energy $\varepsilon^{*}=\left(\mathrm{d} \ln g_{\mathrm{M}}\left(\varepsilon_{\mathrm{F}}\right) / \mathrm{d} \varepsilon_{\mathrm{F} \mid \varepsilon_{\mathrm{F}}=\varepsilon_{\mathrm{c}}}\right)^{-1}$.

The exponent $v$ can be calculated in the case of $(2+\varepsilon)$ space dimensions assuming $\varepsilon$ to be small and using perturbation theory. The $(2+\varepsilon)$ perturbation theory gives $v=\varepsilon^{-1}$; extending this to three dimensions, one obtains $v=1$ [8]. The same value of $v$ is also obtained in the self-consistent theory of localization for noninteracting electrons [47].

The behavior of the conductivity in the region of localized states, i.e., for $g\left(L_{0}\right)=g_{0}<g_{\text {c }}$, is also easily investigated using the asymptotic expression for $\beta(g)$ at small $g$ and the expansion of $\beta(g)$ in the neighborhood of $g_{\mathrm{c}}$. Integrating Equation (1.3.6), we find

$$
\int_{g}^{g_{0}} \frac{\mathrm{d} g}{g \beta(g)}=-\ln \left(\frac{L}{L_{0}}\right) .
$$

Separating the most divergent terms (as $g \rightarrow 0$ and $g \rightarrow g_{c}$ ) in the integral on the lefthand side in Equation (1.3.14), we may write it in the form

$$
\int_{g}^{g_{0}} \frac{\mathrm{d} g}{g \beta(g)}=-\ln \ln \left(\frac{g_{1}}{g}\right)+v \ln \left(\frac{g_{0}-g_{\mathrm{c}}}{g_{\mathrm{c}}}\right)+\varphi\left(g_{0}, g\right) .
$$

The function $\varphi\left(g_{0}, g\right)$ determined by Equations (1.3.19) and (1.3.20) is small compared with the divergent terms explicitly written out in Equation (1.3.20). Using Equations (1.3.19) and (1.3.20), we can find the asymptotic behavior of the conductance $g(L)$ as $L \rightarrow \infty$, i.e., as $g \rightarrow 0$, if the initial value is close to $g_{c}$. We have

$$
g=g_{\mathrm{c}} \exp (-L / \alpha)
$$

where

$$
\alpha=B^{-1} L_{0}\left(\frac{g_{\mathrm{c}}-g_{0}}{g_{\mathrm{c}}}\right)^{-v}
$$

is the localization length on the insulator side of the transition, $B=\varphi\left(g_{\mathrm{c}}, 0\right)$, and $L_{0} \sim l$. This approach predicts that the localization length $\alpha$ diverges at the mobility edge with the same exponent $v$ as that for the correlation length. 
For two-dimensional systems, assuming the function $\beta(g)$ to be smooth and monotonic, we can schematically plot it (Figure 1.4, curve $b$ ). We see that this function is everywhere negative and nonzero. It results from Equation (1.3.6) that $\mathrm{d} g / \mathrm{d} L<0$ for any $L$ so that increasing the system size $L$, we always get to the region of small $g$, where the asymptotic expression (Equation 1.3.7), corresponding to localized states, is valid. Thus, the oneparameter scaling theory of localization leads to the conclusion that for potential scattering in an infinite two-dimensional system, all states are localized [43].

We consider now such Fermi energies for which there exists an interval of $L$, where expression (1.3.9) is valid, i.e., for these $\varepsilon_{\mathrm{F}}$ and at least for some $L$, we have $g_{0} \gg \pi^{-2} \ln (L / l)$. Then the constant of integration is determined by the requirement that Equation (1.3.19) coincides with (1.3.9) in the region of large $g$. It follows that $L_{0}=l$, and Equation (1.3.19) becomes

$$
\int_{g_{0}}^{g} \frac{\mathrm{d} g}{g \beta(g)}=\ln (L / l) .
$$

We note that writing the solution in the form of Equation (1.3.23) with $L_{0}=l$ does not mean that we use Equation (1.3.6) for sizes $L_{0} \sim l$, where the assumption of the oneparameter scaling theory, generally, fails; Equation (1.3.23) is obtained by matching the solution of Equation (1.3.6) with the correct asymptotic expression (1.3.9) in the region $L>l$.

For two-dimensional degenerate electron gas, the Boltzmann conductivity (Equation $1.2 .5)$ is proportional to the Fermi energy, $\sigma_{0} / \sigma_{\min }^{(2)}=\pi^{2} g_{0}=\varepsilon_{\mathrm{F}} / \bar{\varepsilon}$, where $\sigma_{\min }^{(2)}=e^{2} / \pi^{2} \hbar, \varepsilon_{\mathrm{F}}$ is measured from the edge of the intrinsic conduction band in the absence of the random potential, and $\bar{\varepsilon} \simeq 0.3 \hbar / \tau$. At $\tau=$ const the mean free path $l$ depends on the Fermi energy according to

$$
l=l\left(g_{0}\right)=l_{1}\left(\varepsilon_{\mathrm{F}} / \bar{\varepsilon}\right)^{1 / 2},
$$

where $l_{1}=(0.6 \hbar \tau / m)^{1 / 2}$

Equation (1.3.23) determines conductance of the system as a function of its size $L$ and the position of the Fermi level. Explicitly separating in Equation (1.3.26) the most divergent terms as $g \rightarrow 0$ and $g_{0} \rightarrow \infty$, we may write [48]

$$
\ln \ln \left(\left(g_{1}+g\right) / g\right)+\pi^{2} g_{0}+\varphi\left(g, g_{0}\right)=\ln (L / l) \text {. }
$$

Here $g_{1}$ is determined by the asymptotic form of $\beta(g)$ at small $g$ and the function $\varphi\left(g, g_{0}\right)$ determined by Equations (1.3.23) and (1.3.25) is small compared with the first two terms on the left-hand side in Equation (1.3.25) as $g \rightarrow 0$ and $g_{0} \rightarrow \infty$. From Equation (1.3.25) we obtain

$$
g=g_{1}\left\{\exp \left[L / \alpha\left(g, g_{0}\right)\right]-1\right\}^{-1}
$$

where

$$
a\left(g, g_{0}\right)=\exp \left[\pi^{2} g_{0}+\varphi\left(g, g_{0}\right)\right] l
$$


For small $g$, the quantity $\alpha\left(g, g_{0}\right)$ does not depend on $g$ and becomes equal to the localization radius of strongly localized states. Generally, the function $\alpha\left(g, g_{0}\right)$ is the localization length that characterizes the rate of conductance decay with increasing $L$. According to Equation (1.3.27), it depends exponentially on $\pi^{2} g_{0}=\varepsilon_{\mathrm{F}} / \bar{\varepsilon}$; for large $\varepsilon_{\mathrm{F}}$ and at a fixed $L$, $\exp \left[L / \alpha\left(g, g_{0}\right)\right]$ is no longer large compared with unity so that the asymptotic behavior at large $L$ is not reached. The critical energy $\varepsilon_{\mathrm{c}}$ (sometimes called the apparent mobility edge) may be defined by the condition $L / \alpha\left(g, g_{0}\right)=1$; taking account of Equation (1.3.27), we may write it in the form

$$
\varepsilon_{\mathrm{c}} / \bar{\varepsilon}=\ln \left[L / l\left(\varepsilon_{\mathrm{c}}\right)\right]-\varphi\left[g_{1} /(e-1), \varepsilon_{\mathrm{c}} / \pi^{2} \bar{\varepsilon}\right] .
$$

As the Fermi level crosses $\varepsilon_{\mathrm{c}}$, we pass from the region where the exponential asymptotic behavior of the conductance is reached and the conductance is very small to the region where this asymptotic behavior is not reached. Because of the strong exponential Fermienergy dependence of $\alpha\left(g, g_{0}\right)$, the energy interval, in which the conductance varies very quickly, though continuously, is very narrow. According to Equation (1.3.28), the position of the apparent mobility edge depends logarithmically on $L$ or on the corresponding inelastic cutoff length. We note that the mobility edge determined by Equation (1.3.28), is located in the region of large $\pi^{2} g_{0}$, where the use of (1.3.9) as the boundary condition to Equation (1.3.6) is justified.

We can illustrate this general argument by a model example, explicitly specifying the function $\beta(g)$. We choose this function in a simple interpolation form

$$
\beta(g)=\ln \left(\frac{g}{g+\pi^{-2}}\right) .
$$

this function has correct asymptotic behavior at large and small $g$.

In this case, straightforward integration of Equation (1.3.26) gives

$$
\psi\left(\frac{g}{g+\pi^{-2}}\right)-\psi\left(\frac{g_{0}}{g_{0}+\pi^{-2}}\right)=\ln (L / l),
$$

where $\psi(z)$ is the function defined by the expression

$$
\psi(z)=\ln \ln (1 / z)-\int_{0}^{z} \frac{\mathrm{d} u}{(1-u) \ln (1 / u)} .
$$

For $g \gg \pi^{-2}$, we have

$$
\psi\left(\frac{g}{g+\pi^{-2}}\right) \simeq-\pi^{2} g-0.5 \ln \left(\pi^{2} g\right)-C,
$$

where $C \simeq 0.13$; for $g<<\pi^{-2}$, the main contribution comes from the first term on the righthand side in Equation (1.3.31). Accordingly, at small $g$ we obtain an expression of the type (1.3.29), in which

$$
\alpha\left(g_{0}\right)=2\left(\pi^{2} g_{0}\right)^{1 / 2} \exp \left(\pi^{2} g_{0}+C\right) l\left(g_{0}\right)
$$


In this case, the energy $\varepsilon_{\mathrm{c}}$ is approximately determined by the equation

$$
\varepsilon_{\mathrm{c}} / \bar{\varepsilon}=\ln \left[L / l_{1}\right]-\ln \left(\varepsilon_{\mathrm{c}} / \bar{\varepsilon}\right)-C .
$$

It follows from Equation (1.3.33) that the conductance falls sharply to very small values in an energy interval of width of about $\bar{\varepsilon}$ near the threshold $\varepsilon_{\mathrm{c}}$. We note that $\sigma(\varepsilon)$ is the energy-dependent conductance that determines the extended-state component of conductance at finite temperatures. If the Fermi level lies within the mobility gap, $\varepsilon_{\mathrm{F}}<\varepsilon_{\mathrm{c}}$, then it follows from Equation (1.1.3) that the temperature dependence of the extended-state conductance has an activated form. The estimation of the integral in Equation (1.1.3) gives an activated behavior of the conductivity with the preexponential factor $\sigma_{\mathrm{a}}=\sigma_{\mathrm{a}}^{(2)}$ determined by the form of the function $\sigma(\varepsilon)$ near the apparent mobility edge $\varepsilon_{\mathrm{c}}$. For $\varepsilon-\varepsilon_{\mathrm{c}} \gg \bar{\varepsilon}$, the function $\sigma(\varepsilon)$ is almost linear

$$
\sigma(\bar{\varepsilon}) \simeq \sigma_{\min }^{(2)}\left(\varepsilon-\varepsilon_{c}\right) / \bar{\varepsilon}
$$

and for $\varepsilon<\varepsilon_{\mathrm{c}}$

$$
\sigma(\bar{\varepsilon}) \simeq \sigma_{\min }^{(2)} \exp [-L / \alpha(\varepsilon)]
$$

where $\alpha(\varepsilon)$ is defined by Equation (1.3.33), in which $\pi^{2} g_{0}$ is replaced by $\varepsilon / \bar{\varepsilon}$. For $k T \gg \bar{\varepsilon}$, the main contribution to the integral in Equation (1.1.3) comes from the region of energies in which the approximation (1.3.35) is valid, and $\sigma_{\mathrm{a}} \simeq \sigma_{\min }^{(2)}(k T / \bar{\varepsilon})$. For $k T<\bar{\varepsilon}$, we obtain $\sigma_{\mathrm{a}} \simeq \sigma_{\min }^{(2)}(\bar{\varepsilon} / k T) \Gamma(k T / \bar{\varepsilon})$, where $\Gamma(z)$ is the Gamma function. In this case, the temperature dependence is related to the shift of the energy region giving the main contribution to the conductance.

Thus, the scaling theory of localization predicts (see Equations 1.3.27, 1.3.33) that the localization length increases very rapidly with $\pi^{2} g_{0}=\varepsilon_{\mathrm{F}} / \bar{\varepsilon}$ and may easily attain very large (macroscopic) values [49]. For realistic system parameters, the condition that the localization length $\alpha(\varepsilon)$ is equal to the sample size $L$ or the dephasing (inelastic scattering) length $L_{\varphi}$ can be easily satisfied. If the localization length $\alpha(\varepsilon)$ is greater than $L$ or $L_{\varphi}$, the system behaves as if the states were extended. This behavior is, however, different from that of standard metals. Indeed, in this case, the conductivity is expected to increase logarithmically with temperature; this logarithmic dependence comes from the weak localization corrections, as discussed in Section 1.2.2. Near the apparent mobility edge, the scaling approach predicts a gradual transition (in a narrow energy interval) from the slow logarithmic temperature dependence (for states above the apparent mobility edge) to the exponential temperature dependence in the region of localized states.

\subsubsection{Some predictions of the scaling theory}

In the above discussion, we considered mostly the case of very low temperatures. Thouless has argued that at nonzero temperatures inelastic scattering breaks quantum interference that produced the localization corrections. Accordingly, for three-dimensional systems, in expression (1.3.14) for the conductivity, one should replace $L$ by the cutoff length 


$$
L_{\varphi}=\sqrt{D \tau_{\varphi}}
$$

where $D$ is the diffusion coefficient and $\tau_{\varphi}$ is the dephasing time (if $L_{\varphi}<L$ ). Thus, we have

$$
\sigma(T)=\frac{e^{2}}{\hbar}\left(\frac{1}{\xi}+\frac{v}{L_{\varphi}(T)}\right)
$$

For electron-electron scattering, the characteristic dephasing time is $\tau_{\mathrm{ee}} \simeq \hbar / T^{p}$. In this case, $L_{\varphi}=\sqrt{D \tau_{\mathrm{ee}}} \simeq \sqrt{\hbar D} T^{p / 2}$. In the region close to the transition, the first term on the righthand side in Equation (1.3.38) tends to zero, and the second term can become dominant.

For two-dimensional systems, at finite temperatures inelastic scattering leads to a cutoff in the divergence, and the cutoff length is $L_{\varphi}$. It follows from Equations (1.3.30), (1.3.32), or (1.2.21) that, for $\xi>L_{\varphi}$, the conductivity acquires a correction, which depends logarithmically on temperature,

$$
\sigma=\sigma_{0}+\frac{p e^{2}}{2 \pi^{2} \hbar} \ln \left(T / T_{0}\right)
$$

Some of the available data on the temperature dependence of the conductivity will be discussed in Sections 1.4 and 1.5 for three-dimensional and two-dimensional systems, respectively.

The scaling theory of localization was extremely successful and was used as a basis for interpretation of transport properties of disordered solids for several decades. This is somewhat surprising, since it was developed for noninteracting charge carriers, whereas the estimations show (see Section 1.2.3) that the energy of Coulomb interaction could be much greater than the kinetic energy so that the interaction effects are expected to be important (especially for the two-dimensional systems corresponding to the critical dimensionality). Accordingly, the effect of charge carrier interactions may not be reduced to the effect of the inelastic cutoff length $L_{\varphi}$, and substantial effort was directed to generalizing the theory to systems of interacting electrons [50-52]. It was suggested [53] that one can incorporate interaction effects phenomenologically into the scaling approach. Indeed, the conclusion of the scaling theory for noninteracting electrons about the localization of all electronic states at arbitrarily weak disorder is based on the asymptotic behavior of the scaling function at large $g$, in particular, on the sign of the constant $A$ in expression (1.3.9). The quantum interference weak-localization corrections give $A>0$. On the other hand, as noted in Section 1.2.2, in the presence of spin-orbit scattering, it is possible that $A<0$, giving $\beta(g)>0$ at large $g$ [35]. In this case, a fixed point $g=g_{\mathrm{c}}$ appears $\left(\beta\left(g_{\mathrm{c}}\right)=0\right)$ implying the possibility of the existence of a metal-insulator transition in two-dimensional systems. However, it was argued that in real systems, in addition to interactions of the spin-orbit universality class, one can find different universality classes (depending on the experimental situation) that result in a change of sign of the quantum correction in expression (1.3.9) [8]. With regard to electron-electron interactions (see Section 1.2.3), it was the shown that strong interactions can, in principle, result in a transition to a metallic state [50, 51]. However, there are certain difficulties in the theory, since the interaction strength appears to be 
divergent at scaling towards strong coupling; this precludes the limiting transition to large scales and low temperatures. In [53], the implication of formal extension of the oneparameter scaling theory for two-dimensional systems was discussed under the assumption that the interactions can be described by setting $A<0$ (i.e., $\beta(g)>0$ at large $g$ ). Using expression (1.3.9) with $A<0$, we can easily integrate Equation (1.3.6) in the metallic region $\left(g>g_{\mathrm{c}}\right)$ and obtain

$$
g(L)=g_{0}+|A| \ln (L / l)
$$

Setting the cutoff length $L_{\varphi} \sim T^{-p}$ and assuming that $L_{\varphi}$ is less than the system size, we obtain, at low temperatures

$$
g(L) \sim \ln \left(T_{0} / T\right)
$$

i.e., we obtain a diverging conductance (vanishing resistance) as $T \rightarrow 0$. This is a nonFermi-liquid state, since the nature of the electronic states is quite different from that of the states in the absence of the interactions which would be localized in this case.

\subsubsection{Minimum metallic conductivity}

Mott has extended the approach of the weak-scattering transport energy to the region near the mobility edge using the following argument [1, 54]. For a degenerate electron gas $(T=0 \mathrm{~K})$, the value of the Fermi momentum $k_{\mathrm{F}}$ decreases as we approach the mobility edge, the corresponding characteristic de Broglie's wavelength $\lambda_{\mathrm{F}}$ increases, and if it becomes comparable to the mean free path $l$, the weak scattering Condition (1.1.2) is violated. As disorder becomes stronger, the states at the Fermi level become Anderson localized; this happens as $k_{\mathrm{F}} l \sim 1$. Using the weak scattering theory, one may write expression (1.2.5) in the form

$$
\sigma=\frac{n e^{2}}{\hbar k_{F}^{2}}\left(k_{F} l\right) .
$$

For a spherical Fermi surface, in the three-dimensional case we have

$$
n=\left(3 \pi^{2}\right)^{-1} k_{\mathrm{F}}^{3}
$$

Ioffe and Regel argued that, in order to apply the standard scattering picture, the electron wavelength $k_{\mathrm{F}}^{-1}$ must be shorter than the mean free path $l$ and both $k_{\mathrm{F}}^{-1}$ and $l$ should exceed the interatomic spacing $a$ (the Ioffe-Regel criterion [55]). Using Equations (1.3.42) and (1.3.43) along with the Ioffe-Regel criterion, we obtain $\sigma=\frac{n e^{2}}{\hbar k_{\mathrm{F}}^{2}}\left(k_{\mathrm{F}} l\right)=\frac{e^{2}}{3 \pi^{2} \hbar} k_{\mathrm{F}}^{2} l \geq \frac{e^{2}}{3 \pi^{2} \hbar} k_{\mathrm{F}} \geq \frac{e^{2}}{3 \pi^{2} \hbar a}, \frac{e^{2}}{3 \pi^{2} \hbar l}$.

Mott has concluded that the conductivity due to electrons in extended states cannot be smaller than the value

$$
\sigma_{\min }=C \frac{e^{2}}{\hbar l}
$$


where $C$ is constant (in this case $\left.C=\left(3 \pi^{2}\right)^{-1}\right)$. The quantity determined by Equation (1.3.44) was called the minimum metallic conductivity. Different values of $C$ suggested in the literature are of the same order of magnitude; a value suggested by Mott is $C=0.026$. Using this value and $l=3 \AA$, one obtains

$$
\sigma_{\min } \simeq 200 \Omega^{-1} \mathrm{~cm}^{-1}
$$

Mott has argued that, since the conductivity cannot be less than that determined by Equation (1.3.44), the conductivity at $T=0 \mathrm{~K}$ jumps abruptly from the value $\sigma_{\min }$ to zero, i.e., the metal-insulator transition is discontinuous (the discontinuity of the function $\sigma(\varepsilon)$ corresponding to dashed line $a$ in Figure 1.5). Mott's concept of the minimum metallic conductivity and discontinuous metal-insulator transition disagrees with the scaling theory of localization, considered above, that predicts a continuous transition (curve $b$ in Figure $1.5)$ as the Fermi level crosses the mobility edge $\varepsilon_{\mathrm{c}}$ for the three-dimensional systems at $T=0$. In the following, we discuss some of the relevant experimental results and some developments of the scaling theory of localization.

Mott's arguments predict the existence of the mobility edge and a discontinuous metalinsulator transition, which can be also applied to two-dimensional systems $[1,54]$. These arguments imply that the conductivity abruptly drops from the value

$$
\sigma_{\min }^{(2)}=C_{2} \frac{e^{2}}{\hbar}
$$

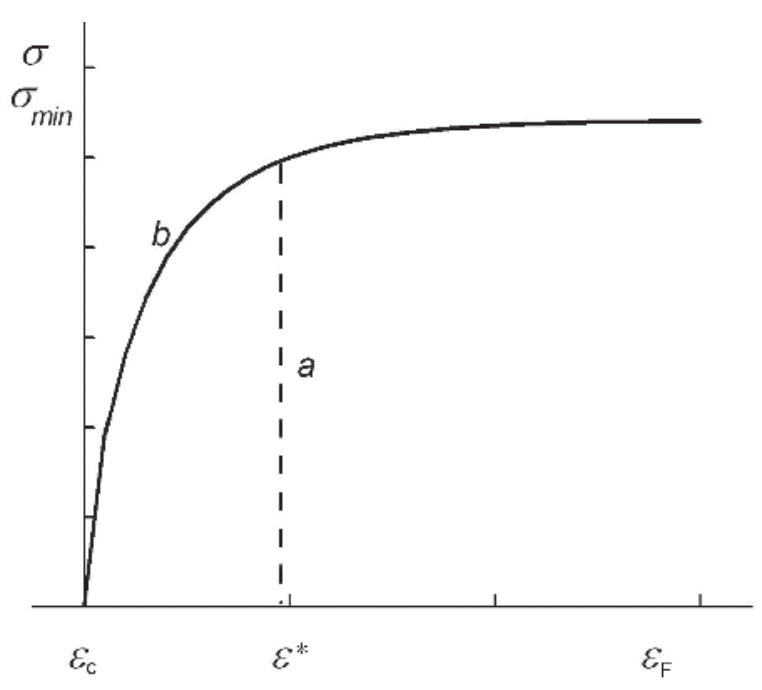

Figure 1.5 Behavior of the conductivity at $T=0 \mathrm{~K}$ near the mobility edge $\varepsilon_{\mathrm{c}}$ (schematic). (a) Discontinuous transition implied by Mott's concept of the minimum metallic conductivity; $(b)$ continuous variation predicted by the scaling theory of localization; the critical region corresponds to the energies $\varepsilon_{\mathrm{c}}<\varepsilon<\varepsilon^{*}$, where the boundary of the critical region $\varepsilon^{*}$ is the energy at which $\sigma\left(\varepsilon^{*}\right)=\sigma_{\min }$ 
to zero; according to Mott's argument and to numerical calculations, $C_{2} \simeq 0.1[1]$. It corresponds to the value of the two-dimensional minimum metallic conductivity $\sigma_{\min }^{(2)} \simeq 2.5 \times 10^{-5} \Omega^{-1}$ (we recall that, in the two-dimensional case, the dimensions of the conductivity and conductance coincide). In the two-dimensional case, the quantity $\sigma_{\min }^{(2)}$ is universal; it contains no length scale.

As noted above, Mott's argument contradicts the conclusion of the one-parameter scaling theory of localization for noninteracting charge carriers, which states that, for twodimensional electron systems, all states are localized and no mobility edge is expected. In a sense, the energy $\varepsilon_{\mathrm{c}}$ separating strongly and weakly localized states might play the role of the mobility edge, since for the states with $\varepsilon>\varepsilon_{\mathrm{c}}$, the localization length $\alpha$ exceeds the system size (or the relevant phase-breaking length) and these states are virtually extended. However, even above the apparent mobility edge, the 'insulator' features of the conductivity behavior corresponding to the behavior of weak-localization corrections with a negative temperature coefficient of the resistivity are expected to persist. On the other hand, a signature of a metallic-type behavior (as, in particular, implied by Mott's argument) is a positive temperature coefficient of the resistivity at low temperatures. In the following sections, we discuss in more detail the results of the experimental studies of the conductivity near the mobility edge for both three-dimensional and two-dimensional systems that seem to require a substantial modification of the traditional approaches, based not only on the concept of the mobility edge, but also on the one-parameter scaling theory.

\subsection{EXTENDED-STATE CONDUCTION IN THREE DIMENSIONS}

\subsubsection{Activated conduction}

For amorphous semiconductors, where the Fermi level lies within the mobility gap, the main contribution to the conductivity at not too low temperatures is provided by electrons in extended states above the mobility edge. In this case, the temperature dependence of the conductivity has an activation form

$$
\sigma=\sigma_{\mathrm{a}} \exp \left(-\frac{\varepsilon_{\mathrm{a}}}{k T}\right),
$$

where $\sigma_{\mathrm{a}}$ is the the preexponential factor and $\varepsilon_{\mathrm{a}}$ is the activation energy. If we assume, in accordance with Mott's concept of the minimum metallic conductivity, that the variation of the conductivity $\sigma(\varepsilon)$ is step-like and the conductivity is zero below $\varepsilon_{\mathrm{c}}$ and $\sigma(\varepsilon) \simeq \sigma_{\text {min }}$ above it (at least within the layer of width of the order of $k T$ near the mobility edge), then from Equation (1.1.3) we obtain $\sigma_{\mathrm{a}}=\sigma_{\min }$ and $\varepsilon_{\mathrm{a}}=\varepsilon_{\mathrm{c}}-\varepsilon_{\mathrm{F}}$, provided that the position of the Fermi level $\varepsilon_{\mathrm{F}}$ with respect to the mobility edge $\varepsilon_{\mathrm{c}}$ is independent of temperature.

A temperature dependence of the conductivity of the activation type (Equation 1.4.1) is a characteristic property both of crystalline (ordered) and disordered semiconductors. Such temperature dependence is related to extended-state conduction due to charge carriers activated to the mobility edge. The preexponential factor provides information about the electronic states and conduction mechanism near the mobility edge. However, it is often problematic to extract information about the behavior of the energy-dependent conductivity $\sigma(\varepsilon)$ from the experimental observations of the activated conduction for several reasons. 
For disordered materials an activated behavior of the type specified by Equation (1.4.1) is usually observed at not too low temperatures, so that electrons in a relatively broad range of energies above the mobility edge contribute to the preexponential factor. With lowering temperature, substantial deviations from Equation (1.4.1) occur, related to the onset of hopping conduction over localized states. To find the behavior of the function $\sigma(\varepsilon)$, one needs to know the conductivity at $T=0 \mathrm{~K}$, i.e., the measurements must be performed at very low temperatures, which is possible only if the Fermi level lies very close to or above the mobility edge.

Next, the preexponential factor can be affected by the variation of the position of $\varepsilon_{\mathrm{F}}$ with respect to the band edge $\varepsilon_{\mathrm{c}}$ with temperature (e.g., due to the statistical shift of the Fermi level or to the shift of $\varepsilon_{\mathrm{c}}$ produced by temperature-dependent disorder). Setting $\varepsilon_{\mathrm{c}}-\varepsilon_{\mathrm{F}}=\varepsilon_{\mathrm{a}}$ $-\zeta T$, we obtain that the activation energy $\varepsilon_{\mathrm{a}}$ is equal to the value obtained by the linear extrapolation of the temperature-dependent difference $\varepsilon_{\mathrm{c}}-\varepsilon_{\mathrm{F}}$ to $T=0 \mathrm{~K}$ and the preexponential factor is multiplied by $\exp (\zeta / k)$; once $\sigma(\varepsilon) \simeq \sigma_{\min }$ near the mobility edge, we obtain

$$
\sigma_{\mathrm{a}}=\sigma_{\min } \exp (\zeta / k)
$$

Experimentally, one can often perform measurements on the same or similar samples with activation energies that differ due to different preparation conditions, doping, or external treatments; e.g., in a-Si:H, the activation energy can be varied in a wide range by prolonged preliminary illumination (the Staebler-Wronski effect) [56]. If the preexponential factor were independent from the activation energy, the series of linear $\ln \sigma-T^{-1}$ plots would extrapolate to the same value as $T^{-1} \rightarrow 0$. For three-dimensional systems, however, the situation, where the conductivity is activated, but the preexponential factors are different from $\sigma_{\min }$ and from each other, is quite common. One of the reasons is that $\sigma_{\min }$ contains the length $l$ (see Equation 1.3.28) that can change when the Fermi level is shifted. Moreover, for numerous disordered semiconductors, the empirical relation known as the Meyer-Neldel rule has been established, relating the preexponential factor $\sigma_{\mathrm{a}}$ and the conductivity activation energy $\varepsilon_{\mathrm{a}}$

$$
\ln \sigma_{\mathrm{a}}=\ln \sigma_{\mathrm{a} 0}+G \varepsilon_{\mathrm{a}}
$$

Equation (1.4.3) has been observed for semiconductor oxides [57], amorphous hydrogenated silicon (a-Si:H) [58], chalcogenide glasses [59], polymers [60], and ionically conducting crystals and glasses [61]. The Meyer-Neldel rule applies to chemically closely related semiconductors and to semiconductors where Fermi level positions vary due to preparation conditions, annealing, etc. Detailed studies of the Meyer-Neldel rule were performed for a-Si:H, where the activation energy could be varied due to the Staebler-Wronski effect. The ubiquitous value of $G$ is about $G \simeq 25 \mathrm{eV}^{-1}$.

Although the Meyer-Neldel rule seems to be an almost universal characteristic of semiconductors and is widely studied, to date there is no universal interpretation of this rule for all materials. Equation (1.4.3) is obtained if the temperature coefficient $\zeta$ is proportional to the activation energy. However, such a dependence does not follow from the conventional picture of the activated conduction; it can be obtained using some specific assumptions on the density of localized states required to produce a linear activation energy dependence of the temperature shift of the Fermi level (the statistical shift) [62]. The calculations of the 
statistical shift of the Fermi energy for some realistic appropriately chosen density-of-states models for a well documented case of a-Si:H were performed [63]. These calculations showed that, indeed, the Meyer-Neldel rule, observed in light-soaking experiments for a-Si: $\mathrm{H}$, can be related to the statistical shift of the Fermi level and the resulting temperature dependence of the difference $\varepsilon_{\mathrm{c}}-\varepsilon_{\mathrm{F}}$.

An implication of the Meyer-Neldel rule is that the preexponential factor for the activated extended-state conductivity is determined not only by the properties of electronic states at the mobility edge, but also by the shape of the density of states in the mobility gap. One more complicating factor is that the parameters of the activated conduction may depend on the presence of large-scale fluctuations. In this case, the band edge $\varepsilon_{\mathrm{c}}$ corresponds to the classical percolation level in a random large-scale fluctuation potential.

Thus, the experimental data on the activated conductivity in disordered semiconductors are in general agreement with the concept of the mobility edge and the minimum metallic conductivity. However, the measurements of the activated conduction of disordered semiconductors, where the Fermi level lies in the region of localized states, do not provide straightforward information on the behavior of the conductivity $\sigma(\varepsilon)$ for energies $\varepsilon$ lying close to the transition. To check Mott's assumption on the minimum metallic conductivity and to obtain reliable information about the features of electronic states and the behavior of the conductivity near the mobility edge, one should use low-temperature measurements in the region of the metal-insulator transition under the conditions where the Fermi level is located in the immediate vicinity of the mobility edge.

\subsubsection{Extended-state conduction near the metal-insulator transition}

The metal-insulator transition was observed in numerous disordered solids where the position of the Fermi level with respect to the mobility edge could be varied. This can be achieved, for example, in doped crystalline semiconductors by varying the impurity concentration and compensation. There also exists a possibility to finely tune the mobility edge by applying magnetic field [64], uniaxial stress [65], or by transmutation doping [66, 67]. This can be also done for amorphous semiconductors (a-Si:Nb [68]) and conducting polymers [69]. Magnetic field causes shrinkage of the impurity ground-state wavefunctions thus increasing localization and shifting the mobility edge upwards. Applying stress admixes more extended impurity excited states, thus stimulating delocalization. The change in impurity concentration $N$ can affect the electronic properties of the system in different ways. For doped semiconductors, the quantity $l$ is expected to be the distance between uncompensated impurities $N^{-1 / 3}$ (see, e.g., [70-72]), and Equation (1.3.44) assumes the form

$$
\sigma_{\min }=C \frac{e^{2}}{\hbar} N^{1 / 3}
$$

As predicted by Mott, transition from an insulator to metallic state may be due to electron correlations (Mott's transition) and is expected to occur as the average spacing between impurities $N^{-1 / 3}$ becomes smaller than the critical spacing $N_{\mathrm{c}}^{-1 / 3}$, where $N_{\mathrm{c}}$ is the critical impurity concentration determined by the condition

$$
N_{\mathrm{c}}^{-1 / 3} a_{\mathrm{B}} \cong 0.25
$$


and $a_{\mathrm{B}}$ is the Bohr radius [2]. Condition (1.4.5) implies that delocalization occurs as the overlap energy becomes comparable to the energy of repulsion between electrons localized on the same impurity. On the other hand, a change in impurity concentration $N$ affects the random potential in the material, thus resulting in a disorder-driven transition (Anderson transition). Generally, a change in $N$ also affects the electron concentration $n$ (related to a shift in the mobility edge $\varepsilon_{\mathrm{c}}$ ), so that the metal-insulator transition is an Anderson-Mott transition, where the effects both of disorder and electron-electron interaction can play an important role.

In accordance with Equation (1.3.18), the variation of the zero-temperature conductivity with impurity concentration can by described by

$$
\sigma(0)=\sigma_{\mathrm{M}}\left(\frac{N-N_{\mathrm{c}}}{N_{\mathrm{c}}}\right)^{v},
$$

where the exponent $v$ is the same as in Equation (1.3.18) and the preexponential factor is $\sigma_{\mathrm{M}} \sim \sigma_{\min }$.

To compare the theoretical predictions on the variation of the zero-temperature conductivity near the metal-insulator transition, one needs to extrapolate the results of finitetemperature measurements of the conductivity to $T=0 \mathrm{~K}$. Such extrapolation is not trivial, in spite of the fact that very low temperatures are used (down to tens of $\mathrm{mK}$ ), since some specific features of the conductivity appear in this temperature range and the results sometimes depend on the extrapolation method used. The standard criterion for a metalinsulator transition implies that the metallic state corresponds to a finite resistivity at $T=0 \mathrm{~K}$, whereas in The insulator state, the resistivity diverges as $T \rightarrow 0 \mathrm{~K}$. As discussed in Section 1.2.1, the classical Boltzmann theory predicts that, for metallic conduction, the temperature coefficient of the resistivity $\mathrm{d} \rho / \mathrm{d} T$ at low temperatures is usually positive, since scattering is enhanced with increasing temperature. Therefore, the condition $\mathrm{d} \rho / \mathrm{d} T$ $>0$ is often considered as a 'signature' of a metal and the condition $\mathrm{d} \rho / \mathrm{d} T<0$ as a signature of an insulating state; accordingly, the condition $\mathrm{d} \rho /\left.\mathrm{d} T\right|_{T \rightarrow 0 K}=0$ is considered as a criterion for a metal-insulator transition. Actually, in the low-temperature range, the temperature dependence of the conductivity near the metal-insulator transition may be nonmonotonic and special care should be taken when describing the conductivity behavior. Moreover, it should be noted that, generally, the $\mathrm{d} \rho /\left.\mathrm{d} T\right|_{T \rightarrow 0 K}=0$ criterion for a metalinsulator transition is not equivalent to the criterion, based on the appearance of the resistivity divergence as $T \rightarrow 0 \mathrm{~K}$ and these criteria give different critical parameters for the metal-insulator transition.

Thus, in order to perform a reliable extrapolation at $T \rightarrow 0 \mathrm{~K}$, one must know the law of the temperature variation of the conductivity in the low-temperature range. For threedimensional systems, in the classical metallic region (for weak disorder, $k_{\mathrm{F}} l>>1$ ), where a description based on the Boltzmann equation may be used, the low-temperature conductivity has the form

$$
\sigma=\sigma_{0}-A T^{s}
$$

where $\sigma_{0}$ is the residual conductivity due to scattering by structural disorder and impurities and $A$ is constant. Additional scattering (by phonons or electron-electron collisions) is usually enhanced with increasing temperature so that both $A$ and $s$ are positive (for 
electron-electron scattering, $s=2$ ). Thus, the conductivity $\sigma$ exhibits a typically metallic behavior, decreasing with increasing temperature.

With decreasing $k_{\mathrm{F}} l$, the disorder becomes stronger and the conductivity decreases, approaching the value $\sigma_{\min }$ determined by Equation (1.4.4). In contrast to Mott's idea, the scaling theory of localization predicts that the conductivity varies continuously in the critical region where $\sigma<\sigma_{\min }$, where the conventional weak-scattering picture does not apply. In this region, quantum interference effects become important. The boundary of the critical region is defined by $\sigma \simeq \sigma_{\min }$ or $\xi \simeq l$ (up to a factor of order unity). Inside the critical region, the conductivity is given by Equation (1.3.38). The temperature-dependent correction describing the quantum interference can be rewritten, taking into account that the diffusion constant $D$ is related to the conductivity by the Einstein relation, $\sigma=e^{2} \mathrm{~d} n / \mathrm{d} \varepsilon_{\mathrm{F}} D$ (for interacting particles, $\mathrm{d} n / \mathrm{d} \varepsilon_{\mathrm{F}}$ may not coincide with the one-particle density of states at the Fermi level). Accordingly, we obtain

$$
\sigma=\frac{e^{2}}{\hbar}\left(\frac{1}{\xi}+v \sqrt{\frac{e^{2}\left(\mathrm{~d} n / \mathrm{d} \varepsilon_{F}\right) T^{p}}{\hbar \sigma}}\right) .
$$

Thus, the temperature coefficient of the metallic conductivity is expected to change its sign as we pass from the classical to the critical region; as noted above, this happens as the value of the conductivity becomes close the minimum metallic conductivity $\sigma_{\min }$, given by Equation (1.3.44).

If the quantum correction is small (in the region of the applicability of the weaklocalization theory) and $p=1$, then $\sigma$ is weakly dependent on temperature and, therefore, varies according to the law

$$
\sigma=a_{1}+b_{1} T^{1 / 2}
$$

where $b_{1}>0$. Since the correlation length $\xi$ diverges as we approach the mobility edge and $a_{1}$ decreases, the second term becomes dominant near the transition, and the temperature dependence of the conductivity in the second term on the right-hand side of Equation (1.4.8) becomes appreciable. If the first term is small compared with the second, this dependence can be found by solving Equation (1.4.8) with respect to $\sigma$ [73]

$$
\sigma=\frac{e^{2}}{\hbar}\left\{\frac{2}{3 \xi}+v^{2 / 3}\left(\frac{\mathrm{d} n}{\mathrm{~d} \varepsilon_{F}}\right)^{1 / 3} T^{p / 3}\right\} .
$$

This is a temperature dependence of the form

$$
\sigma=a_{2}+b_{2} T^{1 / 3}
$$

(for $p=1$ ).

The conductivity near the mobility edge was experimentally studied for numerous disordered solids. We summarize some of the results of the experimental studies of the conductivity near the metal-insulator transition by the example of doped germanium (see, e.g., $[74,75])$. 
1. Figure 1.6 shows the temperature variation of the conductivity for a series of Ge:As samples at varying impurity concentration [75]. A procedure allowing for a reliable extrapolation of the finite-temperature conductivity to $T=0 \mathrm{~K}$ has been described $[75,76]$. The temperature dependence of the low-temperature conductivity for barely metallic samples obeys Equation (1.4.11) with temperature independent $a_{2}$.

The $T^{1 / 3}$ dependence was observed for different doped semiconductors: InSb [64], GaAs $[64,66]$, and Ge [75]. The metal-insulator transition in these experiments was approached by changing both the impurity concentration $N$ and the magnetic field. For samples with concentrations farther from the critical one, the variation of the conductivity can be described by the $T^{1 / 2}$ dependence (Equation 1.4.9); the change from $T^{1 / 2}$ to $T^{1 / 3}$ was observed when the particle energy approaches the mobility edge [64, 66, 77].

2. As the impurity concentration is increased, the temperature coefficient of the resistivity changes sign at some concentration $N_{\mathrm{c}}^{*}>N_{\mathrm{c}}$. According to the theoretical arguments above, this occurs as $\sigma(0)=\sigma_{\min }$ and corresponds to the transition from the critical to the classical region, where $\sigma>\sigma_{\min }$. Thus one can estimate the minimum metallic conductivity by identifying it with the characteristic value of the conductivity at which its temperature

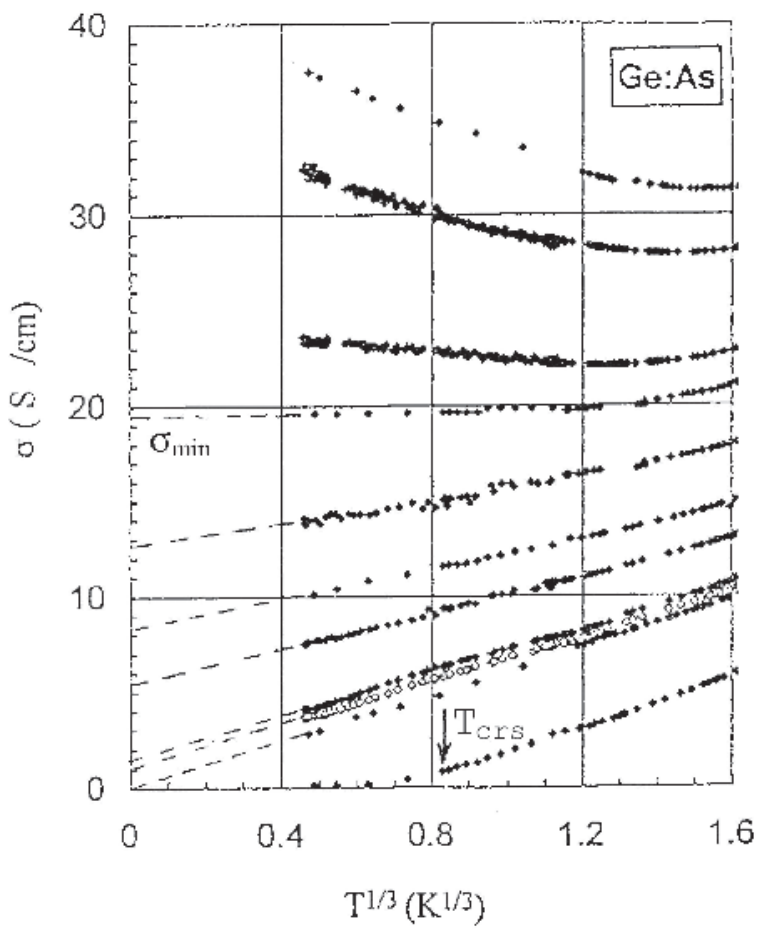

Figure 1.6 Temperature dependence of the conductivity for a series of Ge:As samples plotted as a function of $T^{1 / 3}$. The impurity concentrations are (from top to bottom): $5.38 \times 10^{17} \mathrm{~cm}^{-3}, 5.15 \times 10^{17} \mathrm{~cm}^{-3}$, $4.60 \times 10^{17} \mathrm{~cm}^{-3}, 4.45 \times 10^{17} \mathrm{~cm}^{-3}, 4.17 \times 10^{17} \mathrm{~cm}^{-3}, 3.91 \times 10^{17} \mathrm{~cm}^{-3}, 3.82 \times 10^{17} \mathrm{~cm}^{-3}, 3.58 \times 10^{17} \mathrm{~cm}^{-3}$, $3.56 \times 10^{17} \mathrm{~cm}^{-3}, 3.50 \times 10^{17} \mathrm{~cm}^{-3}, 3.00 \times 10^{17} \mathrm{~cm}^{-3}$. The arrow shows the temperature of the temperature-induced metal-insulator transition (reproduced from [75] with permission from Wiley-VCH) 
coefficient changes sign [75]. The results of this procedure of estimation of the minimum metallic conductivity for different materials with different critical impurity concentrations $N_{\mathrm{c}}$ are shown in Figure 1.7. The order-of-magnitude estimate of $\sigma_{\min }$ agrees with Mott's value and the dependence $\sigma_{\min }\left(N_{\mathrm{c}}\right)$ is well described by Equation (1.4.4) (with different slopes $C$ for $n$ - and $p$-type materials).

3. The experimental studies have shown that a transition from metallic to insulator state is a continuous transition; the values of the zero-temperature conductivity $\sigma(0)$ in the critical region above the mobility edge can be substantially smaller than the minimum metallic conductivity $\sigma_{\min }$, but remain finite, clearly indicating a metallic state. However, it is seen in Figure 1.6 that the $T^{1 / 3}$ dependence is obeyed not only in the critical region $N_{\mathrm{c}}<N<N_{\mathrm{c}}^{*}$ $\left(0<\sigma<\sigma_{\min }\right)$, but for $N<N_{\mathrm{c}}$ in the insulator region close to the transition for $T>T_{\text {crs }}$. Below $T_{\text {crs }}$, the conductivity is low and its temperature dependence is exponential, corresponding to variable range hopping; above $T_{\mathrm{crs}}$ its temperature variation becomes the same as in the critical region on the metallic side of the transition. Thus a crossover to metallic behavior or a temperature-induced insulator-metal transition must occur at $T=T_{\text {crs }}$ as we increase the temperature [78].

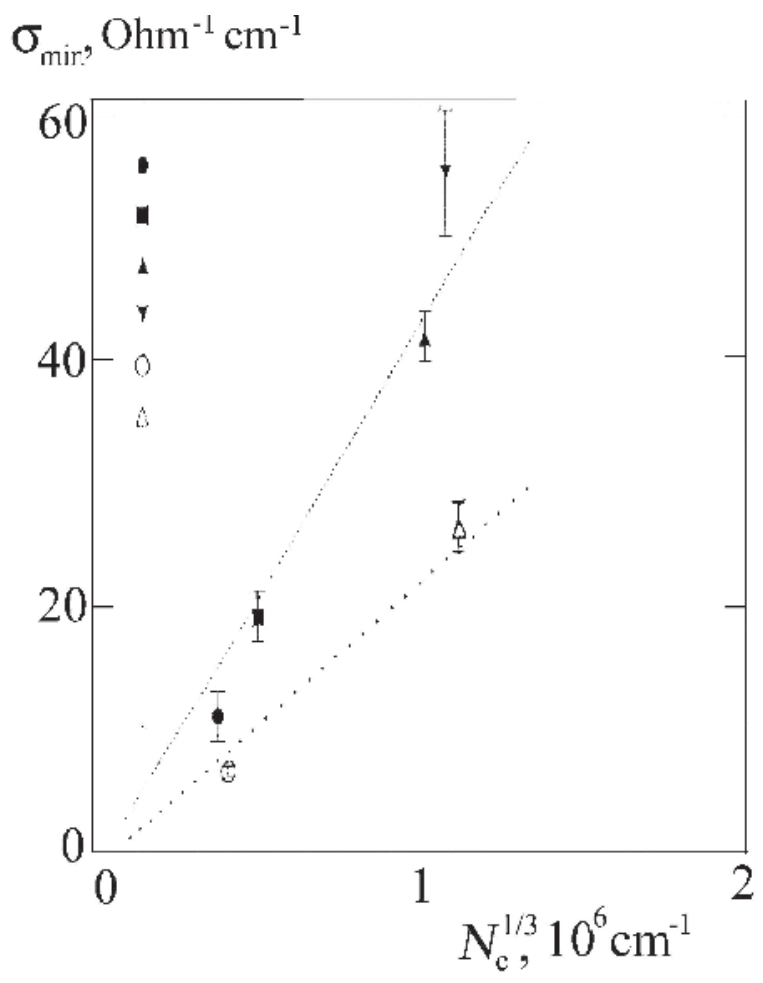

Figure 1.7 Mott's minimum metallic conductivity estimated from the temperature dependence of the conductivity as a function of $N_{\mathrm{c}}^{1 / 3}$ ( $N_{\mathrm{c}}$ is the critical impurity concentration). The slopes are $C=0.12$ for $n$-type and $C=0.06$ for $p$-type materials (reproduced from [75] with permission from Wiley-VCH) 


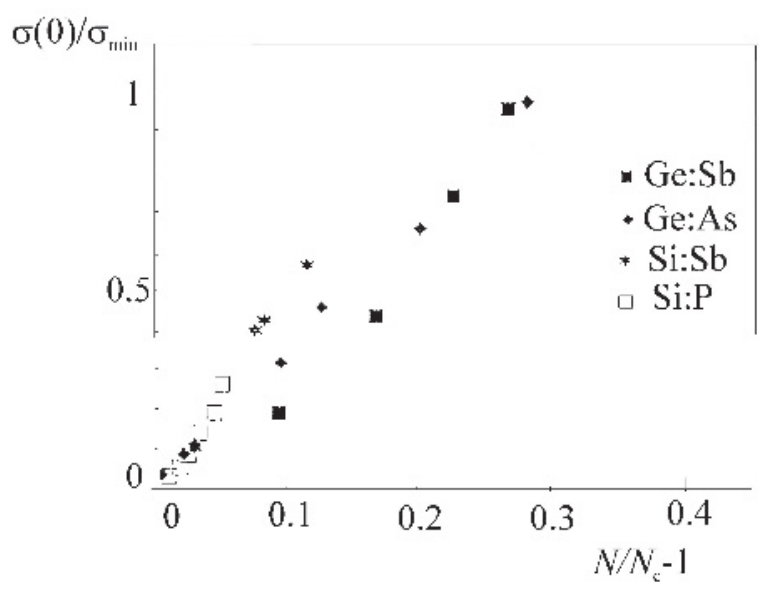

Figure 1.8 Extrapolated values of the normalized zero-temperature $\sigma(0) / \sigma_{\min }$ as a function of impurity concentration for different doped semiconductors (reproduced from [75] with permission from Wiley-VCH)

The variation of $\sigma(0)$ below $\sigma_{\min }$ is usually well described by Equation (1.4.6). It is seen in Figure 1.8 that the data for differently doped $\mathrm{Si}$ and Ge samples fall on a universal curve. This shows the normalization role of $\sigma_{\min }$ in the scaling behavior of zero-temperature conductivity. It is worth noting that theories for interacting charge carriers predict the value $v=1 / 2[79,80]$.

It should be noted that the experimental data on the critical exponent $v$ are controversial; different values have been reported for different materials, ranging from $1 / 2$ to 2 . Sometimes, even the values reported for the same material were different (for instance, in Si:P the values $1 / 2$ [81], 1.3 [82], and 1 [75] were derived). Such discrepancies might be due to the sample properties (in particular, the inhomogeneity in the impurity distribution) and, especially, due to the method of extrapolating the conductivity to zero temperature. The errors may arise due to using the data for samples with concentrations outside the critical region. In [75], the data for a number of Ge and Si samples doped with different impurities (that yielded admittedly different values of $v$ ) were reprocessed using the method described in [76]. The problem of the critical indices does not seem to be completely resolved; indeed, the data obtained for neutron transmutation doped germanium samples with controlled disorder and homogeneity [83] indicate that the critical indices (at least on the insulator side of the metal-insulator transition) may be different, depending on the degree of disorder.

\subsection{APPARENT MOBILITY EDGE AND EXTENDED-STATE CONDUCTION IN TWO-DIMENSIONAL SYSTEMS}

\subsubsection{Experimental studies of the mobility edge in low-mobility two-dimensional systems}

Early experimental studies of the temperature dependence of the two-dimensional conductivity were performed on inversion layers in metal oxide-silicon field-effect transistors 
(MOSFETs). A Si inversion layer is a unique model system for the study of electronic processes in a two-dimensional electron gas, where the carrier density can be varied over several orders of magnitude simply by varying the gate voltage. Moreover, by applying an additional substrate voltage, it is possible to vary the distance between the $\mathrm{Si} / \mathrm{SiO}_{2}$ interface and the two-dimensional layer, thus controlling the magnitude and scale of the random potential fluctuations created by charges in the oxide near the interface [84]. The studies showed that the conductivity has an activation form at not too low temperatures (a transition to hopping was observed with decreasing temperature) and is well described by Equation (1.4.1) at low electron concentrations; at higher concentrations transition to nonactivated metallic-type conduction occurred $[84,85]$. The preexponential factor, which, for short-range potential fluctuations, was close to the minimum metallic conductivity, (Equation 1.3.46), and appeared to be greater than $\sigma_{\min }^{(2)}$ in the presence of long-range fluctuations [85]. These observations were generally consistent with Mott's concept of the minimum metallic conductivity and the existence of the mobility edge.

Subsequent studies of the conductivity behavior in two-dimensional disordered systems (thin metallic films [86], inversion layers in Si MOSFETs [87, 88] have demonstrated the existence of a logarithmic temperature dependence of the conductance in the metallic region above the mobility edge, with a positive temperature coefficient of resistivity, characteristic of the insulating state. Such a dependence agreed with the prediction of the scaling theory of localization and with the concept of the apparent mobility edge separating the regions of strong and weak localization of electronic states (Section 1.3.3); experiments in a magnetic field showed that a substantial logarithmic contribution comes from the correction to the Drude conductivity related to electron-electron interactions in the diffusive regime (Section 1.2.3). To realize the diffusive regime (i.e., to ensure the realization of the condition $l<<L_{\varphi}$ at accessible temperatures), low-mobility samples were used.

\subsubsection{Evidence for a true metal-insulator transition in high-mobility two-dimensional systems}

The measurements of the temperature dependence of the conductivity in high-mobility $\mathrm{Si}$ MOSFETs evidence a real metal-insulator transition in two-dimensional systems [89, 90]. This evidence was corroborated by studies of the temperature dependence of the conductivity in similar Si MOSFETs [91], as well as by measurements on $p$-type $\mathrm{Si} / \mathrm{SiGe}$ [92] and $n$-type $\mathrm{Si} / \mathrm{SiGe}$ [93] heterostructures, $p$-AlGaAs [94, 95], $n$-AlGaAs [96], and $n$-AlAs [97] structures.

We briefly summarize the experimental observations for two-dimensional high-mobility systems.

1. A metallic behavior (positive temperature coefficient of the resistivity) is observed down to the lowest accessible temperatures at charge carrier concentrations $n$ exceeding some critical concentration $n_{\mathrm{c}}$. Below this critical concentration, the behavior of the resistance is insulating, thus indicating that a metal-insulator transition occurs in two dimensions (Figure 1.9). Near the critical concentration, the temperature variation of the resistivity may be nonmonotonic; at $n \simeq n_{\mathrm{c}}$ the resistivity is of the order of the quantum unit of resistance, $h / e^{2} \sim 25.6 \mathrm{k} \Omega$ and is almost independent of temperature (the separatrix in Figure 1.9). 


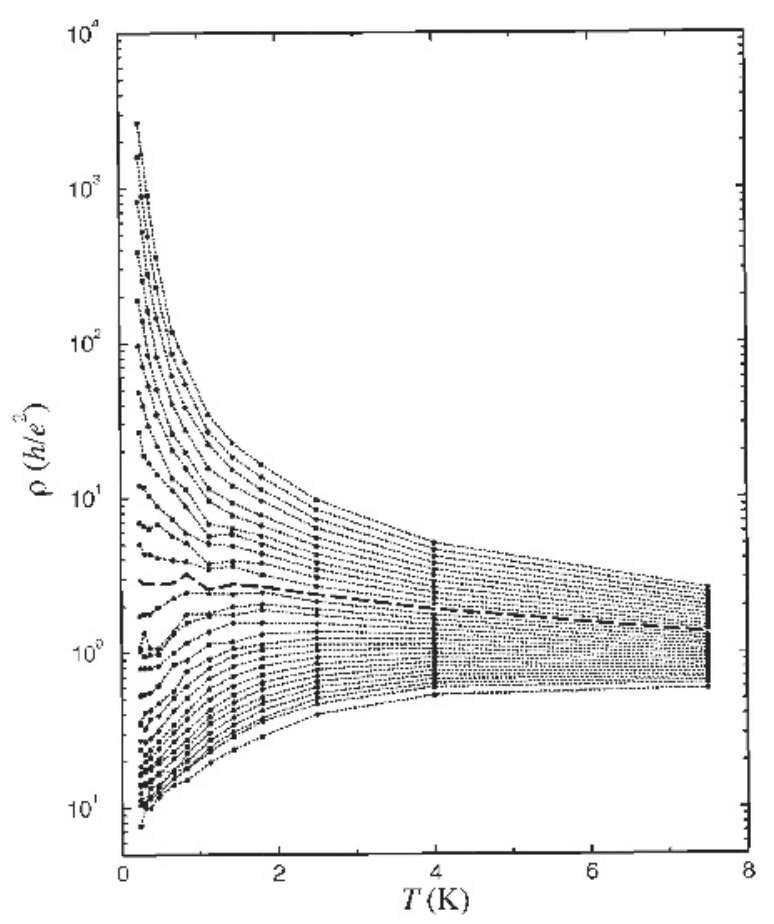

Figure 1.9 Resistivity (in units of $h / e^{2}$ ) of Si MOSFET as a function of temperature for electron concentration varying from $7.12 \times 10^{10} \mathrm{~cm}^{-2}$ to $13.7 \times 10^{10} \mathrm{~cm}^{-2}$ (from top to bottom). The critical density for the metal-insulator transition is $9.6 \times 10^{10} \mathrm{~cm}^{-2}$, indicated by the dashed line (reproduced with permission from [90]; Copyright 1995 by the American Physical Society)

2. Below a temperature of about $2 \mathrm{~K}$, the resistivity exponentially decreases with temperature according to the law

$$
\rho(T)=\rho_{0}+\rho_{1} \exp \left(-\left(T_{0} / T\right)^{p}\right)
$$

where $p \simeq 1$ [98]. The parameter $\rho_{1}$ varies linearly with concentration and vanishes at the transition. The temperature $T_{0}$ is sample dependent and increases with concentration [95], $T_{0} \propto|\delta n|^{q}$, where $\delta n=n-n_{\mathrm{c}}$ and $q \simeq 1$. For high-mobility structures, the resistivity decreases with temperature by about an order of magnitude, whereas in the insulating region the resistivity increases sharply with decreasing $T$.

3. The resistivity for each particular sample (at not too low temperatures) may be scaled using a single scaling parameter $T_{0}$ (Figure 1.10). The resistivity data are reduced into two branches, insulating for $n<n_{\mathrm{c}}$ and metallic for $n>n_{\mathrm{c}}$. The scaling parameter $T_{0}$ has a critical behavior around a critical concentration $n_{\mathrm{c}}$ and decreases upon approaching the critical electron concentration.

4. The magnetoresistance in a weak perpendicular field is negative [91, 98], which indicates the quantum interference contribution to the conductivity of the two-dimensional metallic state. 

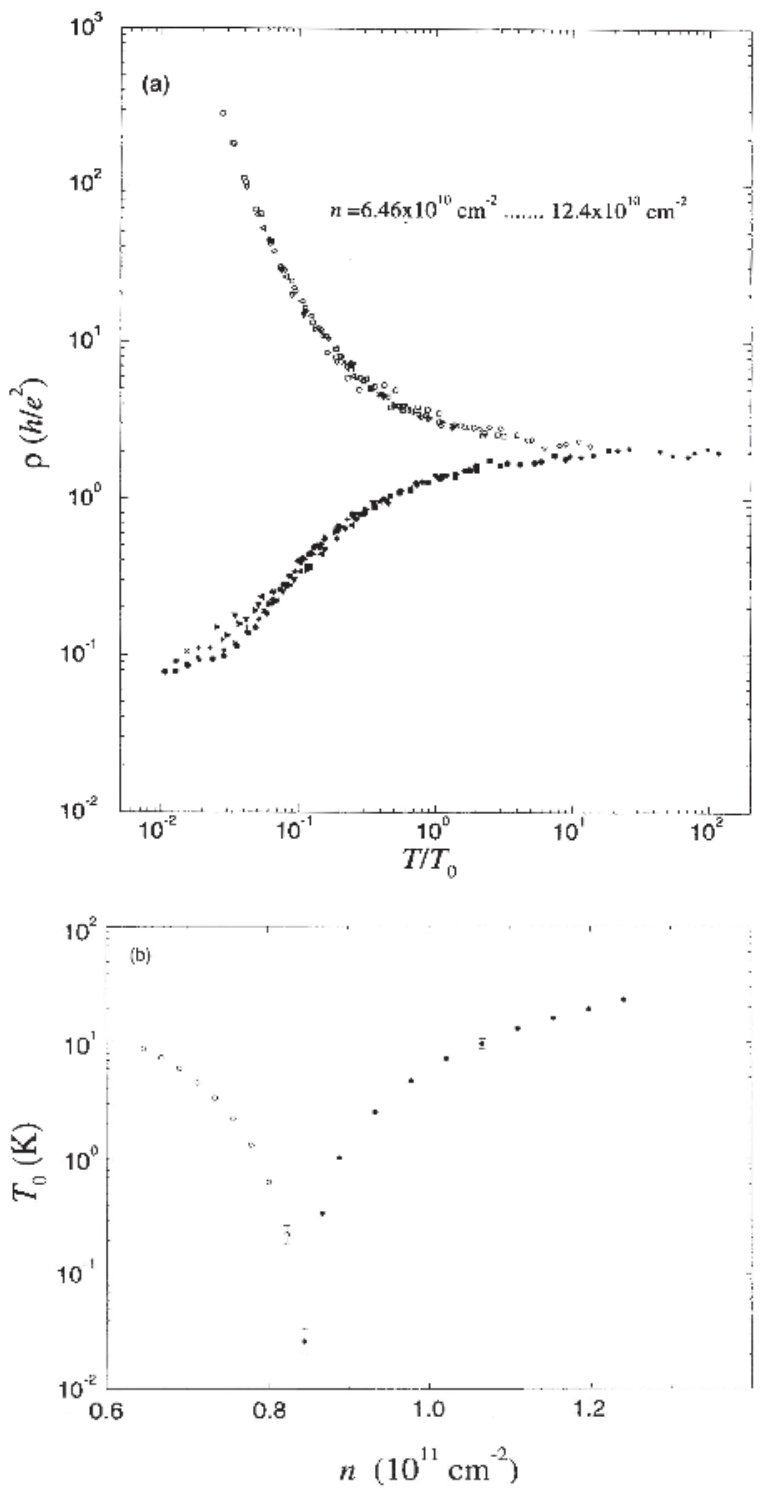

Figure 1.10 Temperature scaling of the resistivity (a) and scaling parameter $T_{0}$ vs electron density (b). Open symbols correspond to the insulating side of the transition and closed symbols to the metallic one (reproduced with permission from [90]; Copyright 1995 by the American Physical Society)

These features are in obvious disagreement with the predictions of the scaling theory of localization and are difficult to explain using the standard Fermi-liquid theory. Kravchenko et al. [90] argued that these results are evidence for the existence of a true metal-insulator transition in two dimensions. Indeed, scaling behavior is one of the signatures of a phase transition [99], suggesting the existence of a true metallic state in a high- 
mobility two-dimensional system. A substantial drop of resistivity on the metallic side of the transition (by an order of magnitude) at low temperatures is, however, difficult to explain using a conventional theory. The difference between the results for high-mobility and low-mobility systems can be related to the higher mobility combined with higher charge carrier effective masses; under these conditions the effect of carrier-carrier interactions is enhanced.

The possibility that carrier-carrier interactions would lead to a qualitative modification of the behavior predicted by the theories for noninteracting electrons was discussed in [50-52], where it has been argued that for weak disorder and arbitrarily strong interactions, a two-dimensional system scales toward a state with finite nonzero conductivity with lowering temperature (as discussed in Section 1.3.4, the rigorous proof of this statement encounters some difficulties). A phenomenological introduction of the interaction effects into the scaling theory of localization also shows that a metallic state that can exist in this case in an unconventional non-Fermi-liquid-type state whose existence is due to electron-electron interactions. Anyhow, a consistent theory that describes the interplay of disorder and interactions near the metal-insulator transition is still lacking.

\subsubsection{Evidence against a true metal-insulator transition in two-dimensional systems}

A different point of view is that the specific features of the low-temperature transport that seem to indicate a true quantum metal-insulator transition (positive metallic-type temperature coefficient of the resistivity in the apparently metallic region, scaling, etc.) can basically be understood in the context of the conventional 'classical' Fermi-liquid-type theory. A number of experimental results confirm this point of view.

(i) Metallic-type temperature coefficient of the resistivity was observed for densities about 30 times greater than the critical density (for resistivities about 100 times smaller than the resistivity at the transition) [100]. In this region, quantum interference and interaction effects are known to be small compared with the large variation of the Drude resistivity; this suggests that the main $g(T)$ dependence in the metallic phase is not due to quantum interference and interaction effects.

(ii) A correlation between the existence of several conducting bands, i.e., of several parallel gases of charge carriers (e.g., holes of different bands in GaAs/AlGaAs heterostructures), and the metallic behavior was studied [101]. The observed metal-insulator transition in the hole gas depended only slightly on the concentration of charge carriers in the split-off band (the concentration in the parallel channel); if it were determined by the interactions, one would expect that increasing the parallel concentration would screen the interactions between holes and suppress the metallic phase in the hole gas. On the other hand, a clear correlation between the resistance and the measured interband carrier scattering was established for the structures under study [101].

(iii) Some experiments have been successfully interpreted in the framework of normal Fermi-liquid behavior of charge carriers on the metallic side of the transition (using the Hartree-Fock approximation), including weak-localization corrections [100, 102, 103]. In [102], magnetoresistance measurements were used to extract the logarithmic corrections to the Drude conductivity in the 'metallic' phase of a high-quality two-dimensional GaAs hole 
system at low temperatures. It has been shown that the phase coherence is preserved in the metallic regime with evidence for normal Fermi liquid behavior and that the anomalous exponential decrease in resistivity with decreasing temperature in the metallic phase was not due to quantum interference or strong interaction effects.

Thus, the experiments indicate that the fundamental difference in the behavior of the conductivity of a two-dimensional gas in the metallic and dielectric regions, corresponding to the metal-insulator transition and the 'anomalous' metallic-type behavior may not be due to the appearance of a new quantum state of a system of strongly interacting charge carriers. An alternative 'quasiclassical' explanation is that anomalous behavior of the resistivity can be described using a Fermi-liquid-type approach. To describe the metallic behavior in this approach, a number of studies used an assumption of temperature-dependent charge carrier scattering.

\subsubsection{Temperature-dependent charge carrier scattering}

\section{(a) Origin of the temperature dependence of scattering at low temperatures}

At low temperatures, phonon scattering may usually be neglected in the temperature range where the anomalous metallic behavior is observed; in Si MOSFET two-dimensional electron systems and two-dimensional $n$-GaAs systems, phonon scattering may be disregarded at $T<5 \mathrm{~K}$, whereas in two-dimensional $p$-GaAs systems, it can play a role at $T>1 \mathrm{~K}$, being responsible for the observed nonmonotonic variation of the conductivity at intermediate temperatures $(1 \mathrm{~K}<T<5 \mathrm{~K})$ [104]. It is known that the main scattering mechanisms in two-dimensional inversion layers are scattering by interface roughness, which is known to be important at higher impurity concentrations (as the two-dimensional electron gas is located closer to the interface), and scattering by charged impurity centers (at the $\mathrm{Si} / \mathrm{SiO}_{2}$ interface in Si MOSFETs) [105]. Moreover, in some structures, additional effects affecting the conductivity and scattering are to be taken into account, such as scattering between the split heavy-hole bands (in GaAs/AlGaAs heterostructures), recharging of trap states at the interface [106], freeze-out of free carriers [107], etc.

In this approach, the main factor responsible for metallic conductivity in a twodimensional electron gas is temperature-dependent disorder, arising, in particular, from the temperature dependence of the screening of static disorder. It appeared that the experimental observations of the 'anomalous' metallic properties of two-dimensional electron systems (a metallic variation of the conductivity, a strong decrease in conductivity with decreasing temperature in the metallic region) can be explained in the framework of the theory based on the quasi-classical Boltzmann approach, even disregarding the quantum interference effects [108]. The single-site approximation was used, since usually the condition $k_{\mathrm{F}} l \gg 1$ is satisfied [109], and electron-electron interactions were taken into account through screening described within the random phase approximation; indeed, screening of the interactions with charged impurities (and, generally, with surface roughness) is the key factor that determines such behavior of the system $[107,108,110]$. For elastic impurity scattering, the low-temperature conductivity $\sigma(T)=n e^{2} \tau(T) / m$ is described by $[108,110]$

$$
\sigma(T)=\sigma(0)\left\{1-1.39 C(n)\left(k T / \varepsilon_{\mathrm{F}}\right)-0.81 C^{2}(n) C_{3 / 2}\left(k T / \varepsilon_{\mathrm{F}}\right)^{3 / 2}+O\left(T^{2}\right)\right\},
$$


where $0<C(n)<1$ is a density-dependent form factor and $\sigma(0)=n e^{2} \tau(0) / m$ is the zerotemperature conductivity within lowest-order coupling to the impurities. It has been shown that if higher-order electron-electron interaction terms are taken into account, in particular, in the ballistic regime for $k T<<\varepsilon_{\mathrm{f}}$, i.e., for $\hbar / \tau<<k T<\varepsilon_{\mathrm{f}}$ (see Section 1.2.3, Equation 1.2.25), the leading linear term in Equation (1.5.2) survives [42]. These conditions, as well the conditions for the validity of Equation (1.5.2), are quite difficult to satisfy, and the measured conductivity virtually never has a linear temperature dependence (except at high densities, where the semiclassical random-phase approximation transport theory is accurate).

To explain the main features of the apparent metal-insulator transition in twodimensional systems, a model was suggested, taking into account localized states in the conduction band tail with regard to doubly occupied states in the upper Hubbard band. In this model, the metallic behavior of the resistance was related to the activation of localized electrons to the conduction band, leading to the suppression of nonlinear screening of the disorder potential [124].

Another possibility, discussed in the context of the metal-insulator transition in a twodimensional hole gas in GaAs/AlGaAs heterostructures, is the effect of inelastic Coulomb hole scattering between the two spin-orbit-split heavy-hole bands on the conductivity in the metallic region. It appeared that the temperature dependence of the measured inelastic interband scattering rate obeyed the relation

$$
S(T)=S_{0}+S_{1} \exp \left(-\left(T_{0} / T\right)^{p}\right)
$$

i.e., had exactly the form of Equation (1.5.1) with almost the same value of $T_{0}$. This mechanism requires a certain band structure and is not universal for all structures but the result (Equation 1.5.3) indicates that the activated temperature dependence of the resistance in the metallic regime may reflect the increase in inelastic interband scattering with temperature and does not necessarily imply the existence of an interaction-induced true metallic state.

\section{(b) Extension of the scaling theory}

A more comprehensive theory would require a simultaneous description of the features of quasiclassical scattering and of the localization and interaction quantum corrections. A generalization of the scaling theory of localization to the case of temperature-dependent disorder and temperature-dependent scattering has been given [111]. Following [111], one can assume that the disorder potential generally consists of the two components so that the classical (Drude) conductivity $\sigma_{\mathrm{D}}$ can be represented as

$$
\sigma_{\mathrm{D}}^{-1}=\sigma_{1}^{-1}+\sigma_{0}^{-1}(T)
$$

where $\sigma_{1}$ is the temperature-independent residual conductivity, whereas $\sigma_{0}$ is the temperature-dependent metallic contribution (such that $\mathrm{d} \sigma_{0} / \mathrm{d} T<0$ ). A straightforward generalization of the approach suggested in [43] is the assumption that the conductance, in addition to the dependence on the system size $L$, may also explicitly depend on $T$ due the corresponding dependence of the disorder. Now in Equation (1.3.19) of the scaling theory, 
we have $g_{0}=g_{\mathrm{D}}=\sigma_{\mathrm{D}}, \sigma_{\mathrm{D}}=\sigma[l(T), T]$ and the scaling function is same as in Equation (1.3.19). When $L_{\varphi}<L$, one has to replace $\ln (L / l)$ by $\ln \left(L_{\varphi} / l\right)$ on the right-hand side of Equation (1.3.23). Assuming for simplicity that $g_{\mathrm{D}}=\sigma[l(T), T] \sim l(T)$ and differentiating Equation (1.3.19) with respect to $T$, we obtain

$$
\frac{1}{\beta(g)} \frac{\mathrm{d} \ln g}{\mathrm{~d} \ln T}=\frac{\mathrm{d} \ln L_{\varphi}}{\mathrm{d} \ln T}+\left(1-\frac{1}{\beta\left(g_{\mathrm{D}}\right)}\right) \frac{\mathrm{d} \ln g_{\mathrm{D}}}{\mathrm{d} \ln T} .
$$

Since the phase-breaking time $\tau_{\varphi}$ may depend on disorder, the authors of [106] have set $\tau_{\varphi} \sim T^{-p} \sigma^{2 \gamma-1}$, where $p$ and $\gamma$ are some constants. In addition, $D \sim \sigma$ so that $L_{\varphi}=\sqrt{D \tau_{\varphi}} \sim \sigma^{r} T^{-p / 2}$. Then Equation (1.5.5) takes the form

$$
\left(\frac{1}{\beta(g)}-\gamma\right) \frac{\mathrm{d} \ln g}{\mathrm{~d} \ln T}=\frac{p}{2}+\left(\frac{1}{\beta\left(g_{\mathrm{D}}\right)}-1\right) \frac{\mathrm{d} \ln g_{\mathrm{D}}}{\mathrm{d} \ln T} .
$$

Equation (1.5.6) is supplemented with the 'boundary' condition $g\left(T_{0}\right)=g_{\mathrm{D}}\left(T_{0}\right)$, where $T_{0}$ is the cutoff temperature at which localization effects become negligible, $L_{\varphi}\left(T_{0}\right)=l\left(T_{0}\right)$. Thus one can account for the $g(T)$ dependence, arising both from the phase-breaking processes (the first term on the right-hand side) and the temperature dependence of the classical conductance $g_{\mathrm{D}}(T)$ (the second term).

This approach provides a rather good description of the experimental data in highmobility MOSFETs, including the metallic behavior of the conductivity above some critical concentration $n_{\mathrm{c}}$, the temperature dependence of $\sigma(T)$ near $n_{\mathrm{c}}$, and a nonmonotonic variation of $\sigma(T)$ in a range of concentrations near $n_{\mathrm{c}}$ : a maximum of the conductivity could be the result of a superposition of a temperature dependent scattering and weak localization and interaction effects.

The quasiclassical (Drude) resistivity was assumed to be temperature dependent due to the variation in carrier scattering. One of the reasons for the metallic behavior of the resistivity is the temperature-dependent screening of charged impurity scattering centers at the interface temperature-dependent concentration of charged defects (see Section 1.5.2.1).

A nonmonotonic temperature dependence may be explained by additionally taking account of some classical scattering mechanism giving rise to the insulator-type temperature-dependent contribution to the resistivity related, e.g., to the metallic freeze-out of the free carriers [107] or the quantum localization corrections. Even a simplified classical approach makes it possible to describe (at least qualitatively) the behavior of twodimensional systems near the metal-insulator transition, including the nonmonotonic temperature dependence at concentrations close to the critical one, concentration dependence of the conductivity, scaling properties; a good agreement was attained for high-mobility lowdensity charge carrier gas in Si MOSFETs and GaAs heterostructures. The observed scaling properties of the conductance $\sigma(T, n) \approx \sigma\left(T / T_{0}\right)$, as well as the concentration dependence of the scaling parameter $T_{0}$ [90], can be reproduced using the Boltzmann model [111].

\section{(c) Effects of a large-scale fluctuation potential}

Up to now, we have discussed homogeneous systems in the absence of strong inhomogeneities in the concentration distribution. However, the presence of a fluctuation potential $V(\mathbf{x})$ 
is a characteristic feature of impurity semiconductors [112]; in two-dimensional systems, this potential can be created by ionized impurities (with concentrations of about $10^{12}-$ $10^{13} \mathrm{~cm}^{-2}$ ), which are usually present at the heterointerfaces, creating potential fluctuations in the plane of the two-dimensional charge carrier gas. A large-scale potential can be also intentionally produced by introducing an array of quantum dots with random parameters [113].

The amplitude of the fluctuation potential increases with decreasing electron concentration, as the screening becomes weaker and strongly nonlinear. Due to this, the spatial distribution of electrons becomes highly inhomogeneous; the electrons are concentrated in the regions of lower potential energy (valleys). The conductivity problem for an electron gas in the presence of a fluctuation potential is known to be intimately related to the continuum percolation problem [5]. An electron with energy $\varepsilon$ can move only in the classically accessible regions defined by the condition $\varepsilon>V(\mathbf{x})$. At $T=0 \mathrm{~K}$, the electrons are located in the regions where $\varepsilon_{\mathrm{F}}>V(\mathbf{x})$ forming puddles and the transport is controlled by electron transfer between the puddles. At low $\varepsilon_{\mathrm{F}}$, such regions are isolated; they grow in size with increasing $\varepsilon_{\mathrm{F}}$ until, at some critical $\varepsilon_{\mathrm{F}}=\varepsilon_{\mathrm{c}}$ (percolation threshold), isolated puddles merge together to form an infinite classically accessible region (the infinite percolation cluster). For $\varepsilon_{\mathrm{F}}>\varepsilon_{\mathrm{c}}$, electrons at the Fermi level can travel classically (above the potential landscape) over the entire system. Thus, a percolation-type metal-insulator transition is expected at some critical average charge carrier concentration $n_{\mathrm{c}}$, corresponding to the condition $\varepsilon_{\mathrm{F}}=\varepsilon_{\mathrm{c}}$. Such a transition was discussed by Efros for modulation-doped heterostructures $[114,115]$. The real situation is somewhat more involved than the simple classical continuum percolation problem, since the form of the fluctuation potential depends on the carrier concentration due to nonlinear screening [5]. Nonlinear screening results in substantial variation (flattening) of the potential in the valley regions filled by electrons.

Experimental studies of conductance in low-carrier concentration MOSFETs indicate a substantial role that can be played by the fluctuation potential in such systems. Thus, the correlation between $n_{\mathrm{c}}$ and the quality of the sample and the nonlinearity of current-voltage characteristics in unexpectedly weak electric fields were observed [116, 117]. These results could be explained using the classical percolation approach. At concentrations below the critical concentration $n_{\mathrm{c}}$, metallic puddles in a two-dimensional hole gas are separated by potential barriers and the energy $\varepsilon_{\mathrm{a}}$ of activation to the percolation level is equal to the difference between the percolation level $\varepsilon_{\mathrm{c}}$ and the Fermi level. In an electric field $E$, the barrier height is decreased by $e E L$, where $L$ is the puddle dimension. If the energy $e E L$ becomes equal to the activation energy, the barrier vanishes and the conductivity grows abruptly, leading to highly nonlinear $I-V$ curves ('breakdown'). Since the cluster dimension diverges near the threshold, the critical electric field for the breakdown appears to be low.

For energies only slightly exceeding the percolation level $\varepsilon_{\mathrm{c}}$, large classically accessible regions are connected by constrictions corresponding to saddle points of the potential landscape. If the Fermi level lies close to the percolation level (either slightly above or below it), the conduction is expected to be controlled by the constrictions for which the saddle point energies $\varepsilon_{\mathrm{QC}}$ are close to $\varepsilon_{\mathrm{c}}$. In the regions of constrictions, a purely classical picture of conduction may, however, be incomplete; it must be supplemented by including the possibility of quantum effects such as tunneling through the constrictions between the puddles.

Thus, tunneling through the constrictions can play an important role, even for a large-scale potential. For energies just above the percolation threshold, the width of the 
constrictions of the infinite cluster may be comparable to the de Broglie wavelength [118121]. Such narrow channels are usually rather short and the constrictions represent quantum point contacts (QPC), corresponding to saddle points of the large-scale potential. Quantum tunneling through these saddle points is a ballistic process. For degenerate electron gas, the conductance of such a QPC can be expressed using the Landauer formula

$$
\begin{aligned}
G\left(\varepsilon_{\mathrm{F}}, T\right) & =\frac{2 e^{2}}{h} \int \mathrm{d} \varepsilon\left(-\frac{\partial f_{F}(\varepsilon)}{\partial \varepsilon}\right) \mathrm{T}(\varepsilon) \\
& =\frac{2 e^{2}}{h} \frac{1}{1+\exp \left[\varepsilon_{\mathrm{QC}}-\varepsilon_{\mathrm{F}} / k T\right]} .
\end{aligned}
$$

Here, $f_{\mathrm{F}}(\varepsilon)$ is the Fermi-Dirac distribution function and $\mathrm{T}(\varepsilon)$ is the transmission through the QPC. Expression (1.5.7) was used in $[118,119]$ with $\mathrm{T}(\varepsilon)=\theta\left(\varepsilon_{\mathrm{QC}}-\varepsilon_{\mathrm{F}}\right)$. It should be noted that using (1.5.7) implies that for $\varepsilon_{\mathrm{QC}}<\varepsilon_{\mathrm{F}}$, the temperature dependence of the conductance is such that $\mathrm{dG}\left(\varepsilon_{\mathrm{F}}, T\right) / \mathrm{d} T<0$, and the resistance of a QPC has an activated contribution.

Due to the randomness of the values of $\varepsilon_{\mathrm{QC}}$ for different QPCs, the problem can be reduced to the classical bond percolation problem [3,5]. By Equation (1.5.7), at $T=0 \mathrm{~K}$, the conductance $G\left(\varepsilon_{\mathrm{F}}, 0\right)$ of a QPC is close to $2 e^{2} / h$ if $\varepsilon_{\mathrm{QC}}<\varepsilon_{\mathrm{F}}$ and $G\left(\varepsilon_{\mathrm{F}}, 0\right)=0$ if $\varepsilon_{\mathrm{QC}}>\varepsilon_{\mathrm{F}}$. We identify the puddles with sites and QPCs with bonds and assume that two puddles (sites) are bonded if $\varepsilon_{\mathrm{QC}}<\varepsilon_{\mathrm{F}}$ (the bond is 'open') and they are not bonded (the bond is 'closed') if $\varepsilon_{\mathrm{QC}}>\varepsilon_{\mathrm{F}}$. For a given distribution of the values of $\varepsilon_{\mathrm{QC}}$, on increasing $\varepsilon_{\mathrm{F}}$, we increase the number of open bonds until at some $\varepsilon_{\mathrm{F}}=\varepsilon_{\mathrm{c}}$ the infinite percolation cluster of conjugated open bonds appears. Neglecting the resistances of the puddles and assuming that the conductivity is controlled by QPCs, we obtain that the conductivity of the system is determined by the conductivity $G\left(\varepsilon_{\mathrm{c}}, 0\right)=0$ of the critical QPC (or of the QPCs if there are several QPCs that control the resistance in the percolation path). The percolation threshold $\varepsilon_{\mathrm{F}}=\varepsilon_{\mathrm{c}}$ corresponds to the metal-insulator transition at $T=0 \mathrm{~K}$ : for $\varepsilon_{\mathrm{F}}>\varepsilon_{\mathrm{c}}$, the density of the percolation cluster increases and the zero-temperature conductance varies as $\left(\varepsilon_{\mathrm{F}}-\varepsilon_{\mathrm{c}}\right)^{t}$, where $t \simeq 1.3$ is the conductivity index $[5,122]$. The conductance as a function of the electron density was numerically calculated for a simple $20 \times 20$ square array of QPCs assuming a uniform distribution of the QPC energies. The results of the calculation appeared to be in good agreement with the behavior of the low-temperature resistivity observed near the transition [118].

At finite temperatures, an important feature of the model [118] is that, according to Equation (1.5.7), the resistance of a conducting QPC with $\varepsilon_{\mathrm{QC}}<\varepsilon_{\mathrm{F}}$ increases exponentially with temperature (saturating as $T \rightarrow 0 \mathrm{~K}$ ), whereas the conductance of an insulating QPC increases exponentially. At high temperatures near the transition $\left(\left|\varepsilon_{\mathrm{QC}}-\varepsilon_{\mathrm{F}}\right|<<k T\right)$, the resistances of the QPCs on both sides of the transition are almost equal. As the temperature is lowered, the difference between the QPC resistances with $\varepsilon_{\mathrm{QC}}$ lying on different sides of the transition increases exponentially. Indeed, the resistance of systems on the insulating side of the transition determined by the critical QPCs with $\varepsilon_{\mathrm{F}}<\varepsilon_{\mathrm{QC}}$ grows exponentially. On the other hand, the behavior of the resistance of metallic samples controlled by conducting QPCs with $\varepsilon_{\mathrm{QC}}<\varepsilon_{\mathrm{F}}$ is more complicated and is related to the structure of the percolation cluster. If we disregard the variation in the structure of the percolation cluster, the tempera- 
ture dependence of the resistance of the system is controlled by the temperature dependence of the resistance of conducting QPCs. It is expected to increase (typical metallic behavior) due the increase in the resistance of a conducting QPC with temperature determined by Equation (1.5.7)

$$
R\left(\varepsilon_{\mathrm{F}}, T\right)=\left(h / 2 e^{2}\right)\left\{1+\exp \left[\varepsilon_{\mathrm{QC}}-\varepsilon_{\mathrm{F}} / k T\right]\right\} .
$$

Equation (1.5.8) has the functional form of Equation (1.5.1), where the parameter $T_{0}$ varies linearly with $\varepsilon_{\mathrm{QC}}-\varepsilon_{\mathrm{F}}$, i.e., with density, and vanishes at the transition, in accordance with the experimental observations. Equation (1.5.8) predicts saturation of the resistance at low temperatures. However, when passing from the resistance of a QPC to the resistance of the system, one should also take into account the variation in the number of bonds that belong to the percolation cluster. This number increases with temperature, since the insulating QPCs with $\varepsilon_{\mathrm{QC}}>\varepsilon_{\mathrm{F}}$ lying close to $\varepsilon_{\mathrm{c}}$ join the percolation cluster. Therefore, at low temperatures, where the resistances of the percolation cluster are weakly temperature dependent, the increase in the density of the percolation cluster may result in the increase in the system conductance. Accordingly, in a range of concentrations near the critical concentration corresponding to the condition $\varepsilon_{\mathrm{F}}=\varepsilon_{\mathrm{c}}$, the temperature dependence of the conductivity may vary nonmonotonically; a monotonic metal-like behavior is regained with increasing $\varepsilon_{\mathrm{F}}$ above the critical value.

Additional confirmation of the important role of random QPCs comes from the studies of a quasi-two-dimensional electron gas in Si MNOS structures with an inversion $n$ channel $[120,121]$. In the mesoscopic structures under study, the percolation cluster consisted of independent parallel paths and the resistance of each path was controlled by a single or a few QPCs. In this case, the shape of the current-voltage characteristic appeared to be sensitive to the variation of the number of QPCs in the optimal current paths. Using the description of a QPC by a parabolic potential, it appeared to be possible to describe the temperature and gate voltage dependences of the resistivity with reasonable accuracy.

\subsection{CONCLUSIONS}

Extended-state transport in disordered solids is an important problem, attracting a lot of attention for many years. Generally, to describe transport processes, an approach that takes into account an intricate interplay of disorder and electron-electron interaction effects is needed. High structural disorder substantially complicates the understanding of transport properties, since the conventional methods and concepts of the transport theory based on the weak scattering concept cannot be directly applied to the materials in question. However, most of the theories proposed for the description of transport are based on different versions of a quasi-classical weak-scattering approach and Fermi-liquid description of electronelectron interaction effects (modified to take account of the correction describing quantum effects). Somewhat surprisingly, such approaches used for the description of transport in extended states are often quite efficient. One example is Ziman's theory of liquid metals, which was successfully applied to describe the resistivity of many simple metals, due, in particular, to the fact that the pseudopotentials for electron interaction with ions are strongly screened. For dirty metals, for which the Fermi level lies deeply in the conduction band, 
the temperature dependence of the low-temperature conductivity determined by quantum interference corrections to the result of the weak elastic scattering theory (Drude conductivity) appeared to be in agreement with experiment.

For extended-state transport near the mobility edge, the kinetic energy, Coulomb interaction energy, and the characteristic disorder energy are often of the same order of magnitude; moreover, as the Fermi level approaches the mobility edge (in the vicinity of the metal-insulator transition), the Wigner-Seitz parameter related to the ratio of the Coulomb interaction energy to the kinetic energy becomes large, thus making it necessary to take interaction effects into account. However, even in this case, the main concepts of the oneparameter scaling theory of localization initially developed for noninteracting electrons (with some modifications) often appear to be a reasonable foundation for the description of transport.

In spite of extensive effort, some of the basic important issues are still not completely resolved. In bulk materials, although the scaling theory of the metal-insulator transition (with regard to interaction effects) adequately describes low-temperature transport in the critical region for numerous doped semiconductors, problems still remain, such as the problem of the critical indices. Moreover, in [123], experimental indications are discussed that, in contrast to the predictions of the scaling theory, the metal-insulator transition in amorphous alloys is discontinuous, implying the existence of Mott's minimum metallic conductivity. For two-dimensional systems, the fundamental problem of the existence of a true metal-insulator transition still remains controversial and different models (discussed in Section 1.5) were suggested that can qualitatively describe the anomalous metallic behavior. Thus, further research, both experimental and theoretical, is needed to elucidate the state of the electron system near the apparent metal-insulator transition.

\section{ACKNOWLEDGEMENT}

This study was supported by the Russian Foundation for Basic Research.

\section{REFERENCES}

[1] N.F. Mott and E.A. Davis, Electronic Processes in Non-crystalline Materials, 2nd edn, Clarendon, Oxford, 1979.

[2] N.F. Mott, Metal-Insulator Transition, 2nd edn, Taylor and Francis, London, 1974.

[3] I.P. Zvyagin, Transport Phenomena in Disordered Semiconductors, Moscow State University Press, Moscow, 1984.

[4] Y. Imry, Introduction to Mesoscopic Physics, University Press, Oxford, 2002.

[5] B.I. Shklovskii and A.L.Efros, Electronic Properties of Doped Semiconductors, Springer, Heidelberg, 1984.

[6] V.L. Bonch-Bruevich, R. Enderlein, B. Esser, R. Keiper, A.G. Mironov, and I.P. Zvyagin, Elektronentheorie ungeordneter Halbleiter, Deutscher Verlag der Wissenschaften, Berlin, 1984.

[7] V.F. Gantmacher, Electrons in Disordered Media, Fizmatlit, Moscow, 2003.

[8] P.A. Lee and T.V. Ramakrishnan, Rev. Mod. Phys., 57, 287 (1985).

[9] N.F. Mott and W.D. Twose, Adv. Phys., 10, 107 (1961). 
[10] E. Abrahams, S.V. Kravchenko, and M.P. Sarachik, Rev. Mod. Phys., 73, 251 (2001).

[11] B.L. Altshuler, D.L. Maslov, and V.M. Pudalov, Physica E, 9, 209 (2001).

[12] P.W. Anderson, Phys. Rev., 109, 1492 (1958).

[13] N.F. Mott, Phil. Mag., 22, 7 (1970).

[14] J.M. Ziman, Phil. Mag., 6, 1013 (1961).

[15] J.M. Ziman, Models of Disorder, University Press, Cambridge, 1979.

[16] W.A. Harrison, Pseudopotentials in the Theory of Metals, Benjamin, Menlo Park, 1966.

[17] M.L. Cohen and V. Heine, in: Solid State Physics, vol. 24, p. 37, Academic Press, New York, 1970; Mott N.F. Phil. Mag. B, 44, 265 (1981).

[18] V.L. Bonch-Bruevich, The Electronic Theory of Heavily Doped Semiconductors, Elsevier, New York, 1966.

[19] I.P. Zvyagin, Fiz. Tverdogo Tela., 6, 2972 (1964).

[20] J.S. Langer and T.S. Neal, Phys. Rev. Lett., 16, 984 (1966).

[21] M.J. Katz, Phys. Rev. A, 140, 1323 (1965).

[22] J.B. Krieger and T. Meeks, Phys. Rev. B, 8, 2780 (1973).

[23] D. Chattopadhyay and B.R. Nag, Phys. Rev. B, 12, 5676 (1975).

[24] J.W. Harrison and J.R. Hauser, Phys. Rev. B, 13, 5347 (1976).

[25] I.S. Shlimak, A.L. Efros, and I.V. Yanchev, Sov. Phys. Semicond., 11, 149 (1977).

[26] A.L. Efros and M.E. Raikh, in: Optical Properties of Mixed Crystals, R.J. Elliott and I.P. Ipatova (eds), Elsevier, New York, 1988, p. 133.

[27] S. Fahy and E.P. O'Reily, Appl. Phys. Lett., 83, 3731 (2003).

[28] V. Venkataraman, C.W. Liu, and J.C. Sturm, Appl. Phys. Lett., 63, 2795 (1993).

[29] C. Michel, P.J. Klar, S.D. Baranovskii, and P. Thomas, Phys. Rev. B, 69, 165211 (2004).

[30] L.P. Gor'kov, A.I. Larkin, and D.E. Khmel'nitskii, Pis'ma Zh. Eksp. Teor. Fiz., 30, 248 (1979) [JETP Lett., 30, 228 (1979)].

[31] B.L. Altshuler, A.G. Aronov, and D.E. Khmel'nitskii, Solid State Commun., 39, 619 (1981).

[32] B.L. Altshuler, A.G. Aronov, and D.E. Khmel'nitskii, J. Phys. C, 15, 7367 (1982).

[33] B.L. Altshuler, D.E. Khmel'nitskii, A.I. Larkin, and P.A. Lee, Phys. Rev. B, 22, 5142 (1980).

[34] P.A. Lee, J. Non-Cryst. Sol., 35-36, 21 (1981).

[35] S. Hikami, A.I. Larkin, and Y. Nagaoka, Prog. Theor. Phys., 63, 707 (1980).

[36] B.L. Altshuler and A.G. Aronov, Zh. Eksp. Teor. Fiz., 77, 2028 (1979) [Sov. Phys. JETP, 50, 968 (1979)].

[37] B.L. Altshuler and A.G. Aronov, Solid State Commun., 30, 115 (1979).

[38] W.L. McMillan and J.M. Mochel, Phys. Rev. Lett., 46, 556 (1981).

[39] R.C. Dynes and J. Garno, Phys. Rev. Lett., 46, 137 (1981).

[40] M. Lee, J.G. Massey, V.L. Nguyen, and B.I. Shklovskii, Phys. Rev. B, 60, 1582 (1999).

[41] H. Fukuyama, J. Phys. Soc. Japan, 48, 2169 (1980).

[42] G. Zala, B.N. Narozhny, and I.L. Aleiner, Phys. Rev. B, 64, 214204 (2001); E.A. Galaktionov, A.K. Savchenko, S.S. Safonov, Y.Y. Proskuryakov, L. Li, M. Pepper, M.Y. Simmons, D.A. Ritchie, E.H. Linfield, and Z.D. Kvon, cond-mat/0402139 (2004).

[43] E. Abrahams, P.W. Anderson, D.C. Licciardello, and T.V. Ramakrishnan, Phys. Rev. Lett., 42, 673 (1979).

[44] D.J. Thouless, Phys. Rev. C, 13, 93 (1974).

[45] D.J. Thouless, Phys. Rev. Lett., 39, 1167 (1977).

[46] L.P. Kadanoff, Physica, 2, 263 (1966).

[47] D. Vollhardt and P. Wofle, Phys. Rev. B, 22, 4666 (1980).

[48] I.P. Zvyagin, Phil. Mag. B, 46, 111 (1983).

[49] A. MacKinnon, Z. Phys. B, 53, 1 (1983).

[50] A.M. Finkel'stein, Zh. Eksp. Teor. Fiz., 84, 168 (1983) [Sov. Phys. JETP, 57, 97 (1983)].

[51] A.M. Finkel'stein, Z. Phys. B, 56, 189 (1984). 
[52] C. Castellani, C. DiCastro, P.A. Lee, and M. Ma, Phys. Rev. B, 30, 527 (1984).

[53] V. Dobrosavljević, E. Abrahams, E. Miranda, and S. Chakravarty, Phys. Rev. Lett., 79, 455 (1997).

[54] N.F. Mott, Phil. Mag., 26, 505 (1972).

[55] A.F. Ioffe and A.R. Regel, Prog. Semicond., 4, 237 (1960).

[56] D.L. Staebler and C.R. Wronskii, Appl. Phys. Lett., 31, 292 (1977).

[57] W. Meyer and H. Neldel, Z. Techn. Phys., 12, 588 (1937).

[58] W.E. Spear, D. Allan, P.G. LeComber, and A. Ghait, Phil. Mag. B, 41, 419 (1980).

[59] K. Shimakawa and F. Abdel-Wahab, Appl. Phys. Lett., 70, 652 (1997).

[60] K. Miyairi, Y. Ohta, and M. Ieda, J. Phys. D, 21, 1519 (1988).

[61] T. Dosdale and R.J. Brook, Solid State Ionics, 8, 297 (1983).

[62] I.P. Zvyagin, J. Non-Cryst. Sol., 97-98, 83 (1987).

[63] H. Overhof, in: Hydrogenated Amorphous Silicon, H. Neber-Aeschbacher (ed.), Solid State Phenomena, 44-46, Scitec Publications, Zurich, 1995, p. 535.

[64] D.J. Newson and M. Pepper. J. Phys. C, 19, 3983 (1986).

[65] T.F. Rosebaum, R.F. Milligan, M.A. Paalanen, G.A. Thomas, R.N. Bhatt, and W. Lin, Phys. Rev. B, 27, 7509 (1983).

[66] K.J. Friedland, A.N. Ionov, R. Rentzsch, Ch. Gladun, and H. Vinzelberg, J. Phys. Cond. Mat., 2, 3759 (1990).

[67] A.G. Zabrodskii, Phil. Mag. B, 81, 1153 (2001).

[68] G. Hertel, D.J. Bishop, E.G. Spencer, J.M. Rowell, and R.C. Dynes, Phys. Rev. Lett., 50, 743 (1983).

[69] M. Reghu, C.O. Yoon, D. Moses, Y. Cao, and A.J. Heeger, Phys. Rev. B, 47, 1758 (1993).

[70] H. Fritzsche, in: Metal Non-metal Transitions in Disordered Solids, L.R. Friedman and D.P. Tumhall (eds), SUSSP, Edinburgh, 1978, p. 190.

[71] G. Biscupski, H. Dubois, O. Laborde, and X. Zotos, Phil. Mag. B, 42, 19 (1980).

[72] E.M. Gershenson, L.B. Litvak-Gorskaya, and G. Ya. Lugovaya, Fiz. Tech. Poluprov., 7, 1284 (1981).

[73] B.L. Altshuler and A.G. Aronov, Pis'ma Zh. Eksp. Teor. Fiz., 37, 349 (1983) [JETP Lett., 37, 410 (1983)].

[74] P. Dai, Y. Zhang, and M.P. Sarachik, Phys. Rev. Lett., 66, 1914 (1991).

[75] I. Shlimak, Phys. stat. sol. (b), 205, 287 (1998).

[76] I. Shlimak, M. Kaveh, R. Ussyshkin, V. Ginodman, and L. Resnick, Phys. Rev. Lett., 77, 1103 (1996).

[77] M.C. Malliepaard, M. Pepper, R. Newbury, and G. Hill, Phys. Rev. Lett., 61, 369 (1988).

[78] I. Shlimak, M. Kaveh, R. Ussyshkin, V. Ginodman, L. Resnick, and V.F. Gantmakher, J. Phys. Cond. Mat., 9, 9873 (1997).

[79] S. Hikami, Phys. Rev. B, 24, 2671 (1981).

[80] W. Sasaki, in: Anderson Localization, T. Ando and H. Fukuyama (eds), Springer, Berlin, 1988.

[81] M.A. Paalanen, T.F. Rosebaum, G.A. Thomas, and R.N. Bhatt, Phys. Rev. Lett., 48, 1284 (1982).

[82] H. Stupp, M. Hornung, M. Lakner, O. Madel, and H.V. Lohneysen, Phys. Rev. Lett., 71, 2634 (1993).

[83] R. Rentzsch, A.N. Ionov, Ch. Reich, V. Ginodman, I. Shlimak, P. Fozooni, and M.J. Lea, Fiz. Tverdogo Tela, 41, 837 (1999).

[84] M. Pepper, Proc. Roy. Soc. Lond. A, 353, 225 (1977).

[85] D.C. Tsui and S.J. Allen, Phys. Rev. Lett., 32, 1200 (1974).

[86] G.J. Dolan and D.D. Osherhoff, Phys. Rev. Lett., 43, 721 (1979).

[87] D.J. Bishop, D.C. Tsui, and R.C. Dyne, Phys. Rev. Lett., 44, 1153 (1980).

[88] M.J. Uren, R.A. Davies, M. Kaveh, and M. Pepper, J. Phys. C, 14, 5737 (1981). 
[89] S.V. Kravchenko, G.V. Kravchenko, J.E. Furneaux, V.M. Pudalov, and M. D'Iorio, Phys. Rev. $B, \mathbf{5 0}, 8039$ (1994).

[90] S.V. Kravchenko, W.E. Mason, G.E. Bowker, J.E. Furneaux, V.M. Pudalov, and M. D'Iorio, Phys. Rev. B, 51, 7038 (1995).

[91] D. Popovic, A.B. Fowler, and S. Washburn, Phys. Rev. Lett., 79, 1543 (1997).

[92] P.T. Coleridge, R.L. Williams, Y. Feng, and P. Zawadzki, Phys. Rev. B, 56, R12764 (1997).

[93] T. Okamoto, K. Hosoya, S. Kawaji, and A. Yagi, Phys. Rev. Lett., 82, 3875 (1999).

[94] M.Y. Simmons, A.R. Hamilton, M. Pepper, E.H. Linfield, P.D. Rose, D.A. Ritchie, A.K. Savchenko, and T.G. Griffiths, Phys. Rev. Lett., 80, 1292 (1998).

[95] Y. Hanein, U. Meirav, D. Shahar, C.C. Li, D.C. Tsui, and H. Shtrikman, et al., Phys. Rev. Lett., 80, 1288 (1998).

[96] Y. Hanein, D. Shahar, J. Yoon, C.C. Li, D.C. Tsui, and H. Shtrikman, Phys. Rev. B, 58, R7520 (1998).

[97] S.J. Papadakis and M. Shayegan, Phys. Rev. B, 57, R15068 (1998).

[98] V.M. Pudalov, JETP Lett., 66, 175 (1997).

[99] Sh. Ma, Modern Theory of Critical Phenomena, Benjamin, Massachusets, 1976.

[100] V.M. Pudalov, G. Brunthaler, A. Prinz, and G. Bauer, Phys. Rev. B, 60, R2154 (1999).

[101] Y. Yaish, O. Prusa, E. Buchstab, Sh. Shapira, G.B. Yoseph, U. Sivan, and A. Stern, condmat/9904324 (1999).

[102] M.Y. Simmons, A.R. Hamilton, M. Pepper, E.H. Linfield, P.D. Rose, and D.A. Ritchie, condmat/9910368 (1999).

[103] V. Senz, T. Heinzel, T. Ihn, and K. Ensslin, G. Dehlinger, D. Grützmacher, and U. Gennser, Phys. Rev. B, 61, R5082 (2000).

[104] S. Das Sarma and E.H. Hwang, Phys. Rev. B, 61, R7838 (2000).

[105] T. Ando, A.B. Fowler, and F. Stern, Rev. Mod. Phys., 54, 437 (1982).

[106] B.L. Altshuler and D.L. Maslov, Phys. Rev. Lett., 82, 145 (1999).

[107] S. Das Sarma and E.H. Hwang, Phys. Rev. Lett., 83, 164 (1999).

[108] S. Das Sarma and E.H. Hwang, Phys. Rev. B, 69, 195305 (2004).

[109] V.M. Pudalov, M.E. Gershenson, H. Kojima, G. Brunthaler, A. Prinz, and G. Bauer, Phys. Rev. Lett., 91, 126403 (2003).

[110] A. Gold and V.T. Dolgopolov, Phys. Rev. B, 33, 1076 (1986).

[111] B.L. Altshuler, D.L. Maslov, and V.M. Pudalov, Phys. stat. sol. (b), 218, 193 (2000).

[112] V.A. Gergel and R.A. Suris, JETP, 84, 719 (1983).

[113] E. Ribeiro, R.D. Jäggi, T. Heinzel, K. Ensslin, G. Medeiros-Ribeiro, and P.M. Petroff, Phys. Rev. Lett., 82, 996 (1999).

[114] A.L. Efros, Solid State Commun., 65, 1281 (1988).

[115] A.L. Efros, Solid State Commun., 70, 253 (1989).

[116] V.T. Dolgopolov, A.A. Shashkin, G.V. Kravchenko, C.J. Emeleus, and T.E. Whall, Pis'ma Zh. Eksp. Teor. Fiz., 62, 162 (1995).

[117] S.V. Kravchenko, D. Simonian, M.P. Sarachik, W. Mason, and J.E. Furneaux, Phys. Rev. Lett., 77, 4938 (1996).

[118] Y. Meir, Phys. Rev. Lett., 83, 3506 (1999).

[119] Y. Meir, Phys. Rev. B, 61, 16470 (2000).

[120] B.A. Aronzon, D.A. Bakaushin, A.S. Vedeneev, A.B. Davydov, E.Z. Meilikhov, and N.K. Chumakov, Fiz. Tech. Poluprov., 35, 448 (2001).

[121] A.B. Davydov, B.A. Aronzon, D.A. Bakaushin, and A.S. Vedeneev, Fiz. Tech. Poluprov., 36, 1241 (2002).

[122] D. Stauffer and A. Aharony, Introduction to Percolation Theory, Taylor and Francis, London, 1992.

[123] A. Möbius and C.J. Adkins, cond-mat/0111017 (2001).

[124] V.I. Kozub and N.V. Agrinskaya, Phys. Rev. B, 64, 245103 (2001). 
